\title{
An Inclusive Survey of Contactless Wireless Sensing: A Technology Used for Remotely Monitoring Vital Signs Has the Potential to Combating COVID-19
}

\author{
MOHAMED BAHACHE ${ }^{1}$, JOEL P. LEMAYIAN ${ }^{2}$, WENJIN WANG ${ }^{3}$ AND JEHAD HAMAMREH ${ }^{2}$ \\ ${ }^{1}$ Department of Electrical and Electronics Engineering, M'sila University, M'sila, Algeria.(email: medbahache@ gmail.com) \\ ${ }^{2}$ Department of Electrical and Computer Engineering, Antalya Bilim University,Antalya, Turkey (e-mail: lemayian.joel@ std.antalya.edu.tr) \\ ${ }^{3}$ Electronic Systems group, Eindhoven University of Technology, Eindhoven, Netherlands (e-mail: wwang@tue.nl) \\ ${ }^{2}$ Department of Electrical and Computer Engineering. Antalva Bilim Universitv.Antalva. Turkev (e-mail: jehad.hamamreh@antalya.edu.tr) \\ ${ }^{2}$ Authors are from WISLAB for Innovations in Wireless Communication Computation laboratory. \\ Corresponding author: Joel P. Lemayian (e-mail: lemayian.joel@std.antalya.edu.tr.)
}

This work is funded by the scientific and technological research council of Turkey (TÜBITAK) under grand $119 \mathrm{E} 392$.

\begin{abstract}
With the Coronavirus pandemic showing no signs of abating, companies and governments around the world are spending millions of dollars to develop contactless sensor technologies that minimize the need for physical interactions between the patient and healthcare providers. As a result, healthcare research studies are rapidly progressing towards discovering innovative contactless technologies, especially for infants and elderly people who are suffering from chronic diseases that require continuous, real-time control, and monitoring. The fusion between sensing technology and wireless communication has emerged as a strong research candidate choice because wearing sensor devices is not desirable by patients as they cause anxiety and discomfort. Furthermore, physical contact exacerbates the spread of contagious diseases which may lead to catastrophic consequences. For this reason, research has gone towards sensor-less or contactless technology, through sending wireless signals, then analyzing and processing the reflected signals using special techniques such as frequency modulated continuous wave (FMCW) or channel state information (CSI). Therefore, it becomes easy to monitor and measure the subject's vital signs remotely without physical contact or asking them to wear sensor devices. In this paper, we overview and explore state-of-the-art research in the field of contactless sensor technology in medicine, where we explain, summarize, and classify a plethora of contactless sensor technologies and techniques with the highest impact on contactless healthcare. Moreover, we overview the enabling hardware technologies as well as discuss the main challenges faced by these systems.
\end{abstract}

INDEX TERMS Channel state information, Frequency modulated continuous wave, Radio frequency signals, Contactless, Wireless, Vital signs, Survey, Overview, wireless Technologies, Wireless sensing.

\section{INTRODUCTION}

$\mathbf{R}$ ecent statistics have shown that the population aged $65+$ is projected to increase from $6.9 \%$ to $12 \%$ and in particular from $15.5 \%$ to $24.3 \%$ in Europe [1]. This population range is usually exposed to suffering from chronic diseases like Parkinson's disease, diabetes, and cardiovascular disease (CVD) [2]. In the USA, for example, $80 \%$ of the elderly population have at least one chronic illness and 50\% have at least two. This list presents the major cause of death around the world, where $30 \%$ of global deaths are caused by CVD each year[1]. Furthermore, this age group has the highest mortality rate in the ongoing Coronavirus (COVID19) pandemic. Therefore, continuous monitoring is needed to continuously observe their vitals. Nevertheless, this care will certainly increase the cost of healthcare [2], which is not available for all patients, especially in many underdeveloped countries.

Recent research has shown that the solution to this challenge is to integrate wireless communication and sensing technology, through the deployment of sensor devices on or 
around the human body to supervise their vital signs, forming what is called wireless body area networks (WBANs) [3]. However, implanted sensors on the patient's body make them uncomfortable and require frequent expert intervention. Moreover, physical contact between the patient and the healthcare provider may lead to the spread of dangerous infections such as the Coronavirus [4].

Thus, a robust alternative preference to the contact body area network related solutions has recently emerged under the name wireless sensing, which uses contactless (devicefree or sensor-less) technology for supervising human vital signs. This choice ensures patient comfort, eliminates physical contact between the patient and the healthcare provider, and overcomes other challenges associated with the use of WBANs such as limited resources, fault tolerance [5], security [6], and others.

Several research studies [7] [8][9][[10] showed that it is possible to supervise and measure a user's vital signs by sending RF signals, processing, and analyzing the reflection from the patient. In this paper, we give an overview of the most recent advances in wireless sensing techniques used in many healthcare applications. More specifically, a classification framework is proposed for categorizing the existing wireless sensing techniques related to contactless technologies. Also, a detailed comparison between these techniques is provided to help facilitate choosing the right technique for a given case or scenario. Moreover, we overview the main challenges faced by these systems as well as enabling technologies.

The remainder of this paper is organized as follows (as can be observed from Fig 11): Section II presents and explains the essential functions related to wireless sensing technologies. Section III overviews the contactless technologies proposed and used in the literature to supervise human vital signs. Section IV discusses the hardware technology available in literature that facilitates contactless sensing research. Section $\mathrm{V}$ talks about the challenges faced by each discussed technology. In section VI we discuss lessons learned and research direction, then we conclude the paper in section VII.

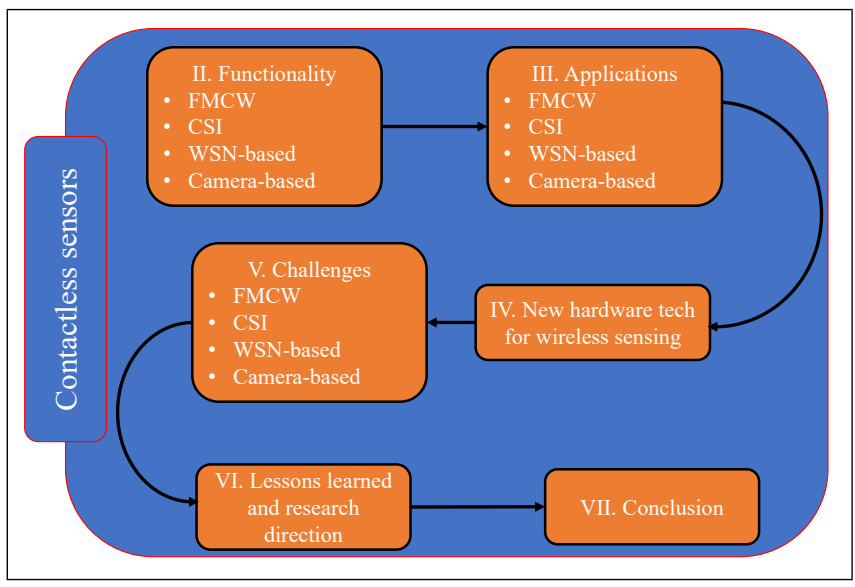

FIGURE 1: The structure of the paper.

\section{A. ABBREVIATIONS AND ACRONYMS}

ADC (Analog to Digital Converter)

AHI (Apnea-Hypopnea Index)

CM-OS(Complementary Metal-Oxide-Semiconductor)

CNN (Conventional Neural Network)

CSI (Channel State Information)

CVD (Cardio Vascular Disease)

DSP (Digital Signal Processor)

ECG (Electro-Cardio-Gram)

EM (Electromagnetic)

FFT (Fast Fourier Transform)

FMCW (Frequency Modulated Continuous Wave)

FSHD (facioscapulohumeral muscular dystrophy)

HMM (Hidden Markov Model)

IF signal (Intermediate Frequency signal)

$\mathrm{KN}$ (K-Nearest)

MIMO (Multiple-Input Multiple-Output)

NMS (None-Maximum Suppression)

NN (Neural Network)

PCA (Principal Component Analysis)

PD (Parkinson's Disease)

REM (Rapid Eye Movement)

RF (Radio Frequency)

RSS (Received Signal Strength)

$\mathrm{Rx}$ (Reception)

SAF (Sub-carrier Amplitude Frequency)

SDP (Sleep Disordered Breathing)

SNR (Signal-to-Noise Ratio)

SNAP (Sensor Network Asynchronous Processor)

STFT (Short Time Fourier Transform)

SVM (Support Vector Machine)

ToF (Time of Flight)

TST (Total Sleep Time)

Tx (Transmission)

WBANs (Wireless Body Area Networks)

WCI (Wireless Channel Information)

WIFI (Wireless Fidelity)

WSN (Wireless Sensor Network)

\section{CONTACTLESS SENSORS: FUNCTIONALITY}

Contactless sensor monitoring is a new technology that is used to supervise vital signs (motion, emotion, sleep, heart rate, respiration rate), without obliging the patient to wear any sensor device.

In most technologies the contactless sensor system broadcasts the wireless signal, then analyzes and processes the reflected signals from the objects. In a typical contactless sensor setup shown in Fig. 22, there are four reflectors (human, wall, bed, and couch), the system begins by suppressing reflected signal from permanently-placed objects (wall, bed, and couch), and focus only on the reflected signal concerning the person under study. Other contactless technologies have the same setup as shown in Fig. 2, where the sensing device is installed away from the subject and is designed to recognise the target from all other objects in the environment. 


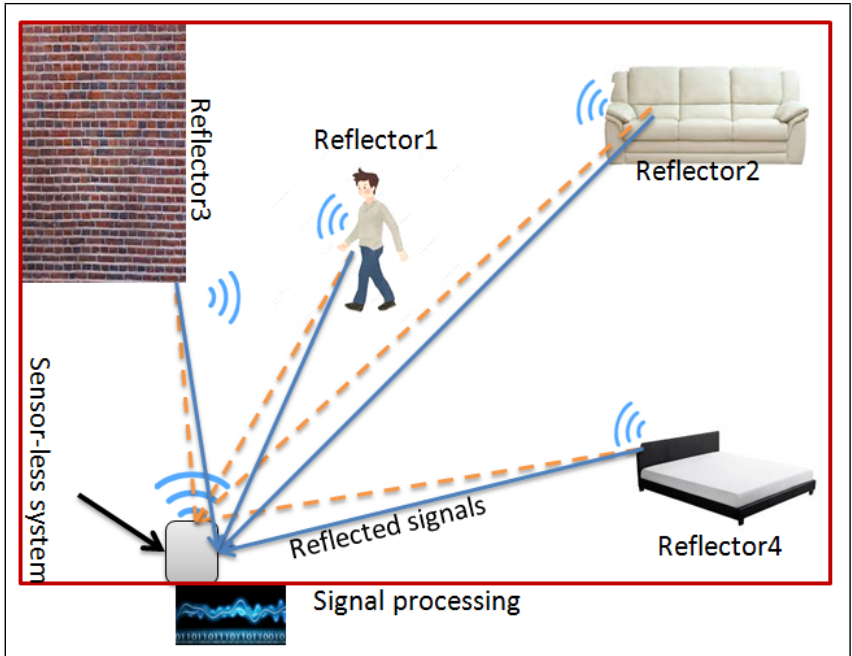

FIGURE 2: Typical contactless sensor monitoring environment

This work classifies patient's vital signs monitoring utilizing contactless sensors into the following main categories:

1) Frequency Modulated Continuous Wave (FMCW) based.

2) Channel state information (CSI) based.

3) Wireless sensor network (WSN) based.

4) Camera-based.

In the following subsection, we will discuss how each of the mentioned technologies operates. We put more emphasis on the FMCW technique since according to our research it is the most commonly used technique and has shown great potential in its applications in future human vital signs monitoring. Moreover, many contactless technologies such as radio frequency (RF) based sensing are based on FMCW technique.

\section{A. FMCW RADAR}

FMCW is used in radar systems for sensing, localizing, and tracking an object in front of it by measuring the range, velocity, and angle of arrival of the reflected wave. In healthcare, this technique sends a special sinusoid signal to a subject then the system analyzes the reflected signal based on its frequency and amplitude to extract information about the observed person [11]|[12]. Here, we present the principle of this technique by answering the following four questions:

First: How do you configure the radar to estimate the range of an object? As shown in Fig. 3 (A), the distance $d$ from the object to the FMCW-radar must be known by the radar. The approximation of intermediate frequency $\omega_{i f}$ is typically used to find the distance $d$.

$$
d=\frac{\omega_{i f}}{\dot{\omega}} \cdot \frac{c_{m}}{2}
$$

where $c_{m}$ is the propagation speed of the electromagnetic (EM) wave in a given medium and $\dot{\omega}$ is the quotient of the chirp's slop frequency [13].
Second: What if there are multiple objects? Fig. 3 (B) depicts the scenario where multiple objects are observed by the FMCW-radar. Therefore, the system must be correctly configured to recognize this scenario.

Third: What is the minimum distance between two objects? Fig. 3 (C) shows two objects very close to each other, the system must be correctly configured to distinguish between one object and two objects which are very close to each other.

Fourth: What determines the furthest distance a radar can see? It is critical that the maximum observable distance by the radar system is determined [14] as shown by Fig. 3(D).

Moreover, we further discuss important features of FMCW-radar in subsequent subsections.

\section{1) Chirp}

It is a signal that exists at the heart of the FMCW-radar. It is considered a sinusoid wave whose frequency increases with time [15], see Fig. 4. As observed from the figure, the chirp starts as a sine wave with the frequency of say, $F C$ and gradually increases its frequency until it reaches the frequency of say $F C+B$, where $B$ is the bandwidth of the chirp, thus the frequency is modulated and this is what is meant by frequency modulated continuous wave (FMCW).

\section{2) Characteristics of a chirp}

The frequency vs. time plot is a convenient way to represent a chirp, as shown in Fig. 5, since the frequency of the Chirp increases linearly with time, it takes the form $S=A t$, where $S$ is the instantaneous frequency of the chirp and $A$ is a constant. In other words a straight line with a given slope. From the figure, the following characteristics of a chirp can be extracted:

1) A start frequency $(F C)$, Bandwidth(B), and duration (Tc).

2) The Slope $(\mathrm{S})$ of the chirp defines the rate at which the chirp ramps up.

In this example, the chirp is sweeping a bandwidth of $4 \mathrm{GHz}$ in $40 \mu \mathrm{s}$, which corresponds to a slope of $100 \mathrm{MHz} / \mu \mathrm{s}$.

\section{3) FMCW radar operation}

After explaining what a chirp is, now we move on to explain how an FMCW-radar works. For this, we consider the simple FMCW-radar shown in Fig. 6(A) with :

- a single Tx antenna,

- a single Rx antenna, and

- a mixer.

A mixer is a three(3) port device with two(2) inputs and one(1) output, as shown by Fig. 6 (B), where for two input sinusoid signals $x_{1}$ and $x_{2}$, there is an output signal $x_{o u t}$, its frequency equals the difference of the two incoming signals' frequencies, and its amplitude also equals the difference of the two amplitudes. For example, if $x_{1}$ and $x_{2}$ are as shown below,

$$
x_{1}=\sin \left(\omega_{1} t+\phi_{1}\right) \text {, }
$$




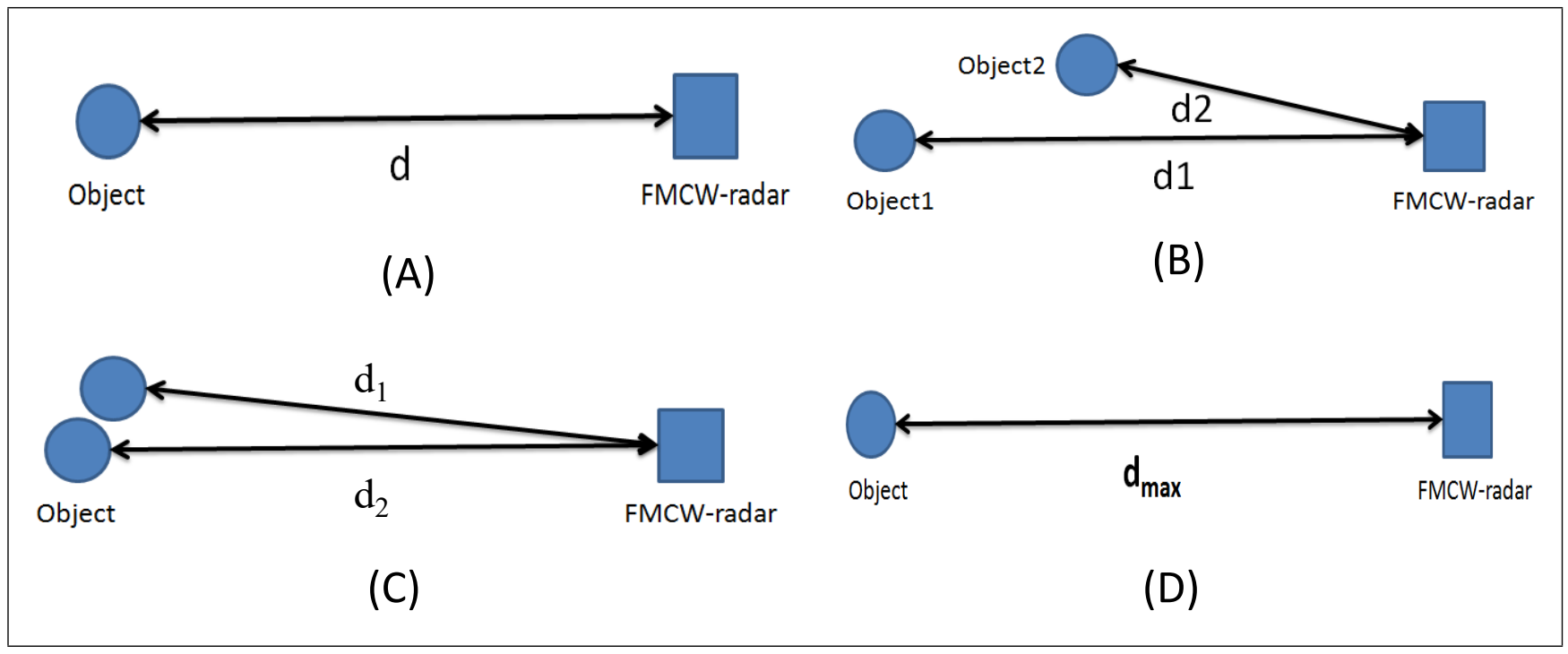

FIGURE 3: (A)Range estimation, (B)Multiple objects, (C)Two objects very close to each other, (D)Maximum observable distance

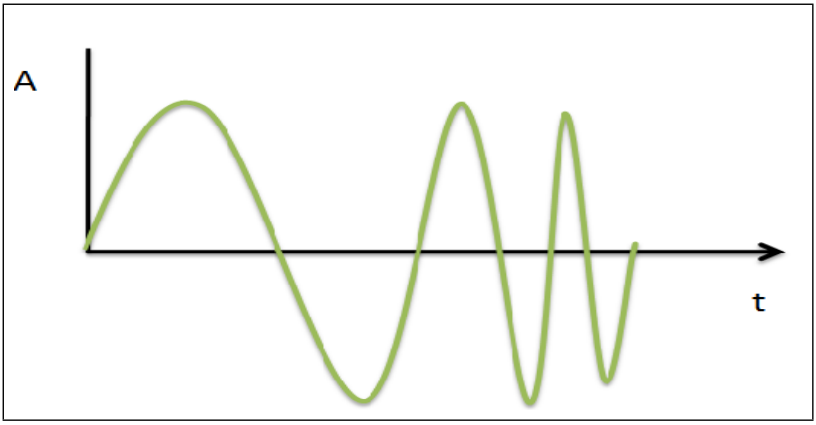

FIGURE 4: Chirp amplitude-time plot

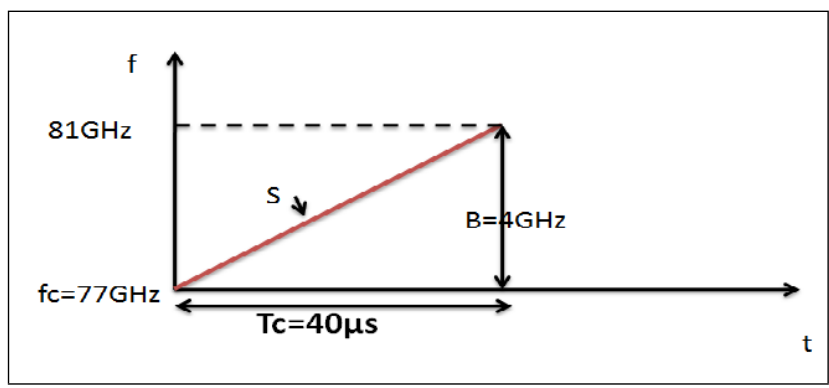

FIGURE 5: Chirp frequency-time plot

$$
x_{2}=\sin \left(\omega_{2} t+\phi_{2}\right)
$$

then $x_{\text {out }}$ is:

$$
x_{\text {out }}=\sin \left(\left(\omega_{1}-\omega_{2}\right) t+\left(\phi_{1}-\phi_{2}\right)\right) .
$$

The FMCW-radar operates according to the following steps:

- First: A synthesizer generates a chirp,

- Second: The chirp is transmitted by the Tx antenna,

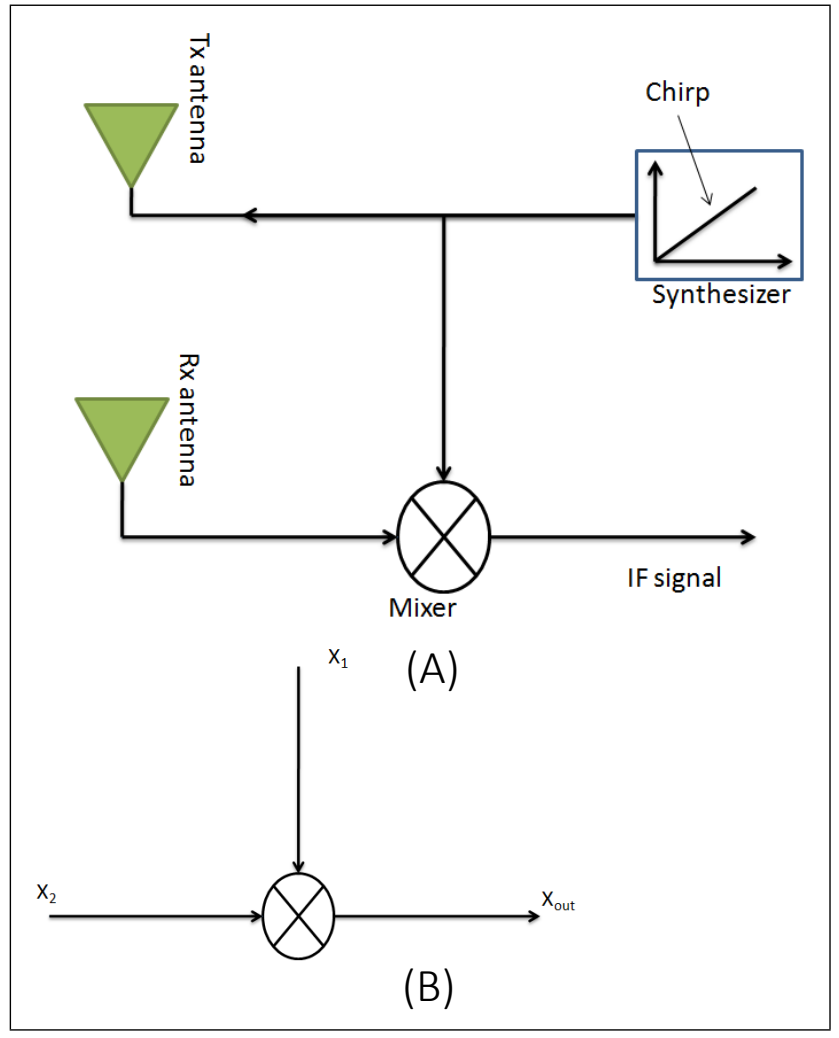

FIGURE 6: (A) Simple FMCW radar, (B) Mixer device
- Third: The chirp is reflected off an object and the reflected chirp is received at the Rx antenna,

- Fourth: The Rx signal and Tx signal are 'mixed' and the resulting signal is called an 'IF signal'. (See the next section for more details). 
4) IF signal

Fig. 7 shows the TxChirp and RxChirp signals (the transmitted and reflected signals from the object), observe that signal from $\mathrm{Rx}$ is a delayed version of signal from $\mathrm{Tx}$, and that IF signal is a straight line with a constant $f$ value.

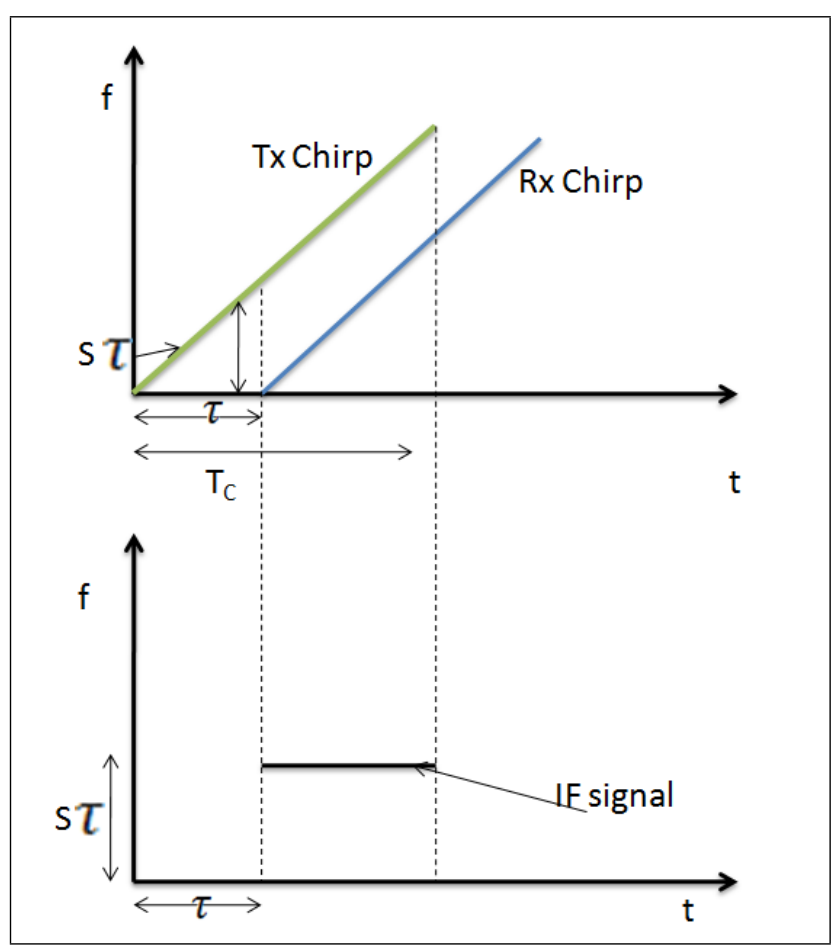

FIGURE 7: IF signal

\section{5) Fourier Transforms (FT)}

Notable properties of the Fourier transform are as follows:

- FT converts a time-domain signal into the frequency domain.

- A sinusoid in the time-domain produces a single peak in the frequency domain (see Fig. 8).

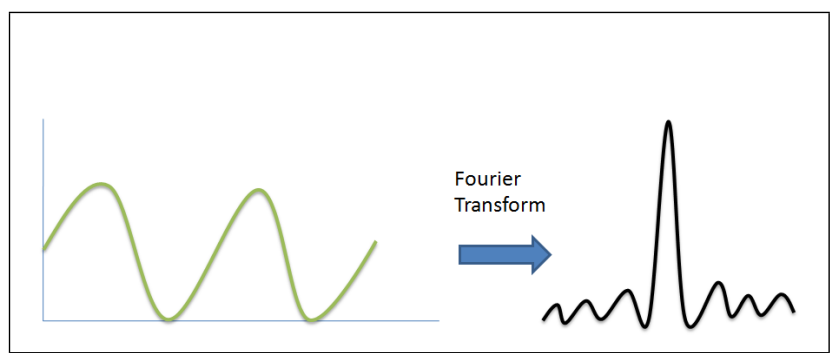

FIGURE 8: Fourier transform

- Within the observation window T below, the orange tone completes 2 cycles, while the blue tone completes 2.5 cycles. The difference of 0.5 cycles is not sufficient to resolve the tones in the frequency spectrum, see Fig. 9

- Doubling the observation window results in a difference of 1 cycle, the tones are resolved in the frequency spectrum, see Fig. 10 .

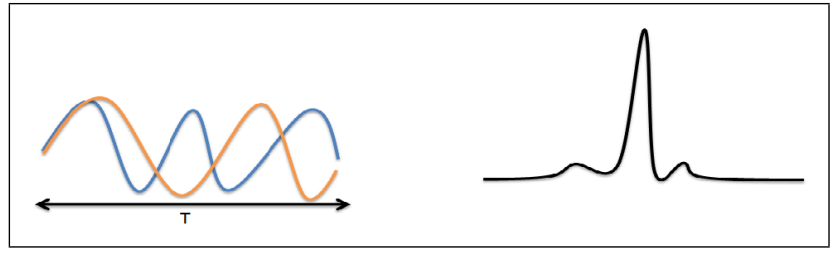

FIGURE 9: Not sufficient observation window

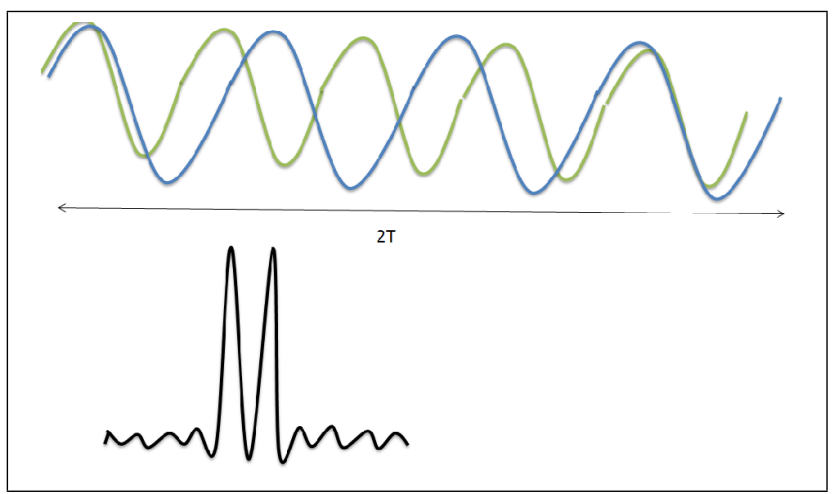

FIGURE 10: Sufficient observation window

- As a result, we can say that a more extended period of observation deduces better resolution.

Since the signals used in an FMCW-radar are sinusoidal, the FT is typically used to estimate parameters such as range, angle, and velocity. Depending on the number of parameters that need to be detected, the FT dimensions are adjusted accordingly [16]. For instance, to detect range and velocity a two-dimensional (2D) FT is required while a fourdimensional (4D) FT is needed to estimate range, velocity, azimuth, and elevation. Hence, the complexity exponentially increases as the dimensions of FT increases.

\section{6) Multiple objects in front of the radar}

Applying the same principle, multiple objects in front of the radar yield multiple reflected chirps at the $\mathrm{Rx}$ antenna, see Fig. 11. A frequency spectrum of the IF signal will reveal multiple tones, the frequency of each being proportional to the range of each object from the radar, see Fig. 12 .

\section{7) Range Resolution in a radar}

To understand how to recognize two objects which are very close to each other, we discuss range resolution in a radar.

1) Range Resolution refers to the ability to resolve two closely spaced objects, see Fig. 13 and Fig. 14

2) The two objects can be resolved by increasing the length of the IF signal, see Fig. 15 and Fig. 16 .

3) Note that this also proportionally increases the bandwidth. Thus intuitively, the greater the Bandwidth, the better the resolution.

4) The range resolution $\left(d_{\text {res }}\right)$ depends only on the Bandwidth swept by the chirp: $d_{r e s}=\mathrm{c} / 2 \mathrm{~B}$ ( $c$ : speed of light, $B:$ Bandwidth). 


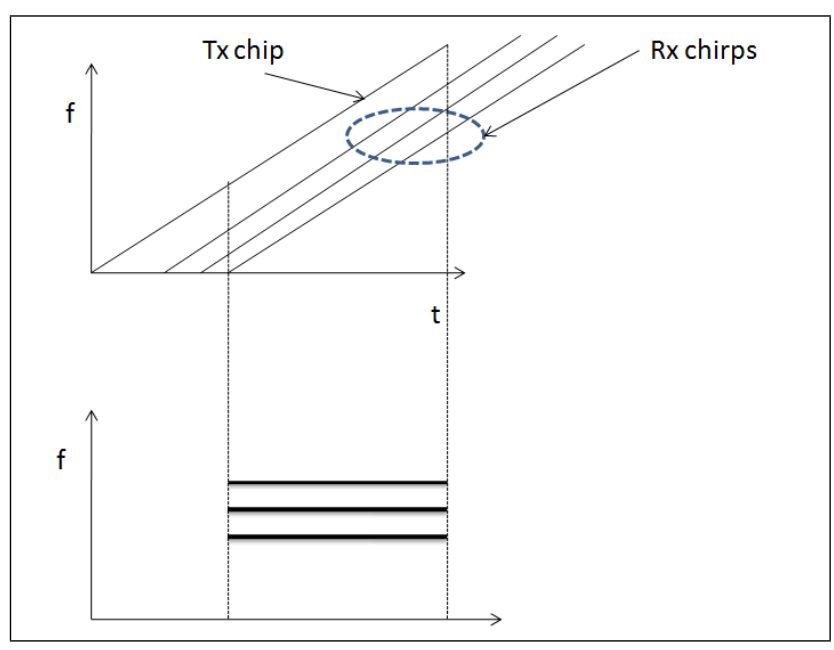

FIGURE 11: Multiple tones in IF signal

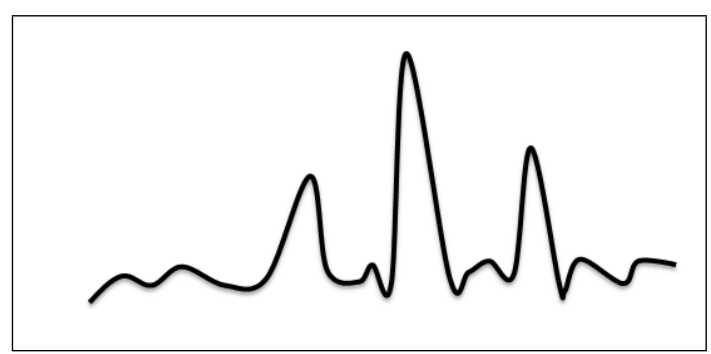

FIGURE 12: IF frequency spectrum

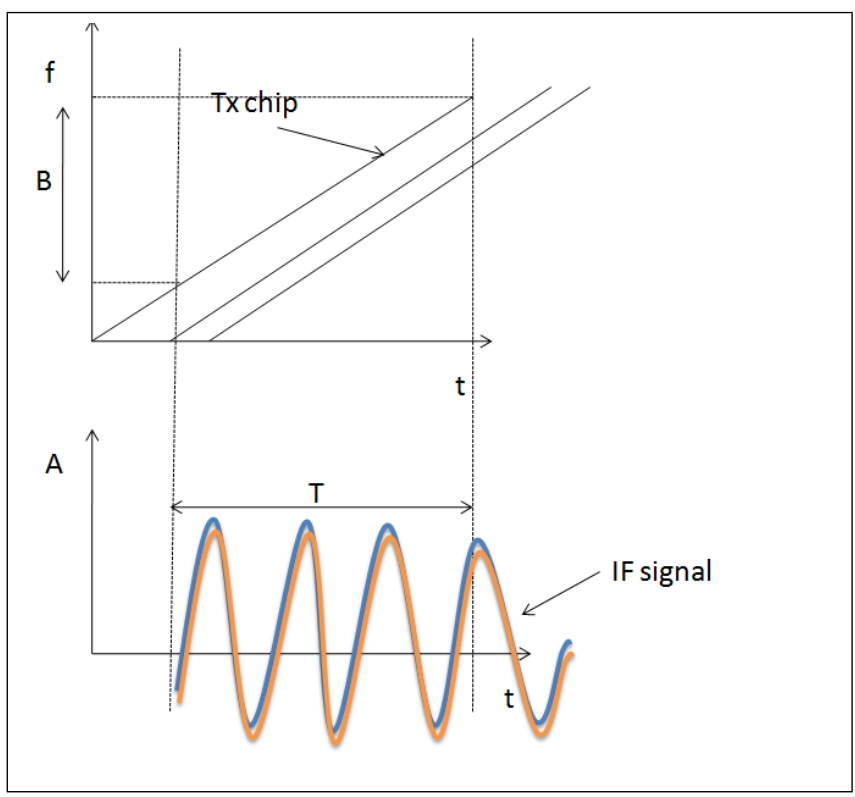

FIGURE 13: Range resolution with insufficient time observation

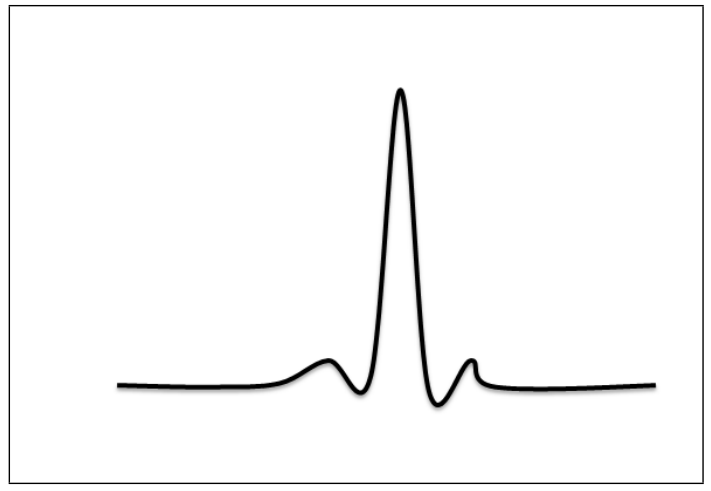

FIGURE 14: IF frequency spectrum(insufficient time observation)

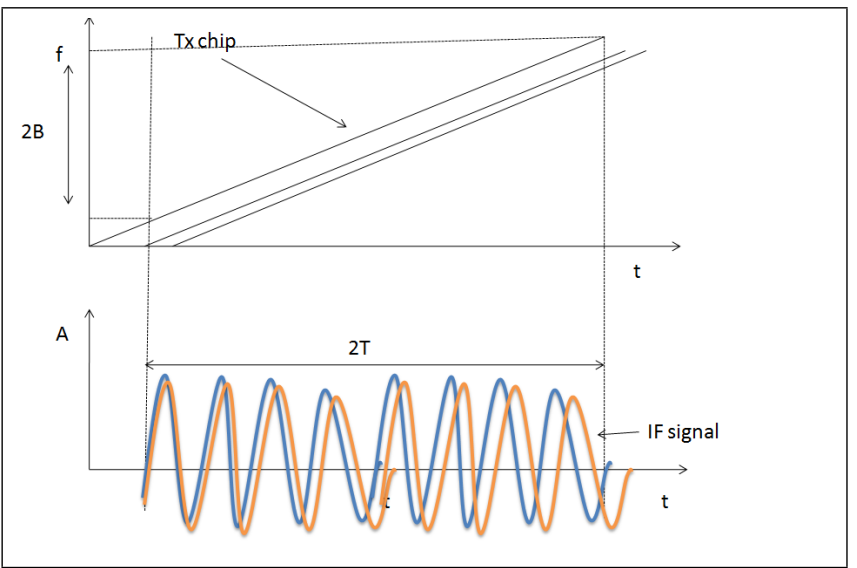

FIGURE 15: Range resolution with sufficient time observation

\section{8) Digitizing the IF signal}

The resultant IF signal is passed through a low pass filter to be digitized by an ADC, then sent to a suitable processor such as DSP that begins by doing the FT to estimate the range, the velocity, and angle of arrival of the object, see Fig. 17

The bandwidth of interest of the IF signal depends on the desired maximum distance: $f_{I F-\max }=\mathrm{S} 2 d_{\max } / \mathrm{c}$, where $S$ is the slope of the chirp.

An ADC sampling rate of Fs limits the maximum range of

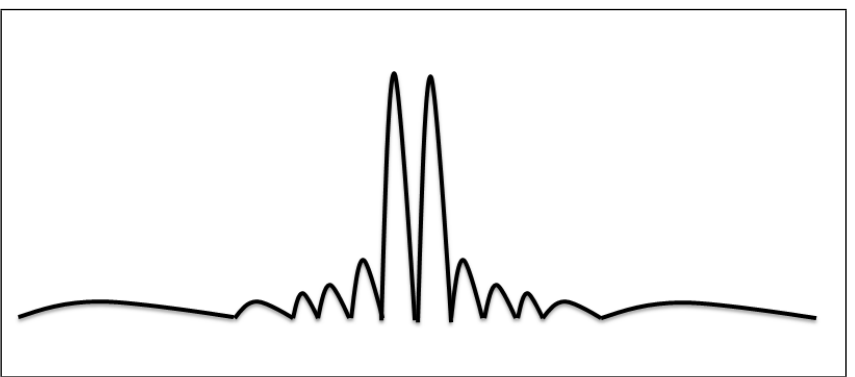

FIGURE 16: IF frequency spectrum(sufficient time observation) 


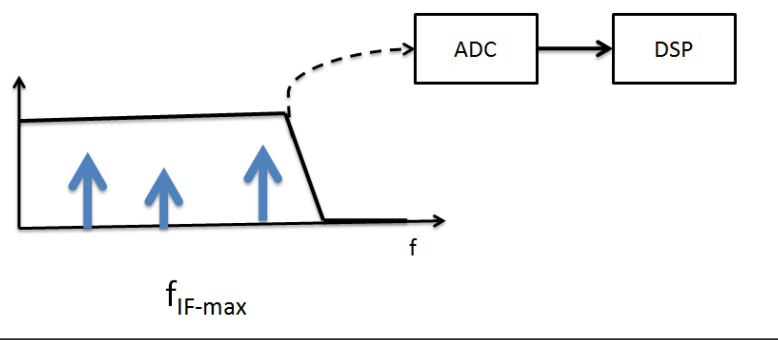

FIGURE 17: IF signal digitizing process

the radar to $: d_{\max }=F_{s} \mathrm{c} / 2 \mathrm{~S}$.

Now we can give the final scheme of our simple radar as seen in Fig. 18, that operates according to the following steps

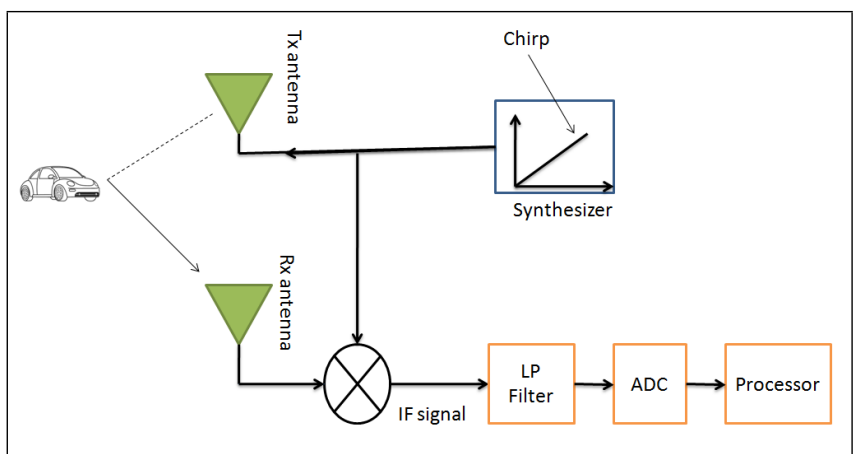

FIGURE 18: Simple FMCW R radar

1) The synthesizer generates chirp,

2) The generated chirp is transmitted. Rx receives delayed versions of this chirp,

3) The IF signal consists of multiple tones, the frequency (f) of each tone being proportional to the distance (d) of the corresponding object,

4) The IF signal is digitized. The ADC must support an IF bandwidth of ( $\left.22 d_{\max } / \mathrm{c}\right)$,

5) An FFT is performed on the ADC data. The location of peaks in the frequency spectrum directly corresponds to the range of objects.

\section{B. CSI-BASED}

Apart from FMCW radar, CSI-based sensing has attracted tremendous attention in detection and interpretation of human activities in healthcare applications such as assisted living and remote monitoring. For instance, due to its pervasive and unobtrusive nature in indoor environments, WiFibased technologies have evolved to a promising residential contactless monitoring technique.

In this contactless technique, advanced signal processing algorithms are used to precisely extract the CSI of the WiFi signal which includes phase shifts, frequency variations and signal levels [17]|[18]. Fig 19 depicts the general structure of CSI-based sensing, where the first step is to capture the signal, then processing the captured signal to extract CSI, and the last step is using machine learning techniques to extract the human behaviour.

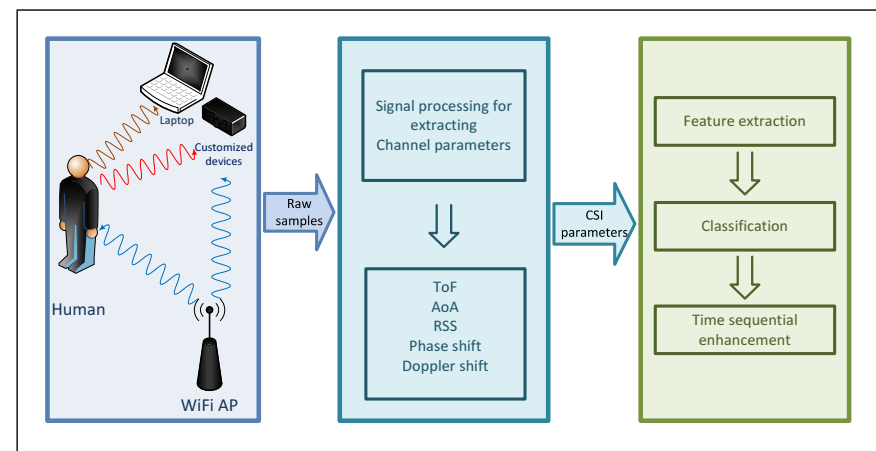

FIGURE 19: The general structure of CSI-based human behaviour sensing

\section{WSN-BASED}

In healthcare, WSNs are a set of sensors nodes randomly positioned and co-operate with each other to monitor, process, and send collected patients' data and their environment to the relevant person [19]. The sensors employ routing protocol for low power and loss network (RPL) for routing packets in a WSN [20].

Emergency services and daily monitoring services in a hospital can be very difficult to manage. Hence, systems which can automate patient monitoring have the ability to enhance patient care. Existing systems include CodeBlue [21], which is a wireless sensor network system which can be deployed at the hospital or at home. The device integrates various medical sensors such as EKG Electrocardiograph, pulse oximeter and EMG.

Moreover, MEDiSN [22] is another system that aims to automate physiological monitoring in different settings. The system comprises of several physiological sensors monitoring the patient and have a temporary memory where information is stored and scripted before transmission. Also, a wireless patient monitoring system developed by Washington University is closely related to MEDiSN [23]. Fig 20 depicts WSN nodes deployed in a hospital and home setting.

\section{CAMERA-BASED}

This system uses cameras to capture the images of subjects (for example their faces) and uses intelligent algorithms to decipher information (for example, pain, confusion, fear, etc). In other cases, thermal camera are used, which have the ability to register the temperature of a subject. In medicine, these special cameras are deployed in quarantine facilities to continuously monitor people and help recognise people with infectious diseases by monitoring their temperature [24][25].

Moreover, in advanced camera-based vital signs monitoring RGB-thermal image sensors are used to monitor not 


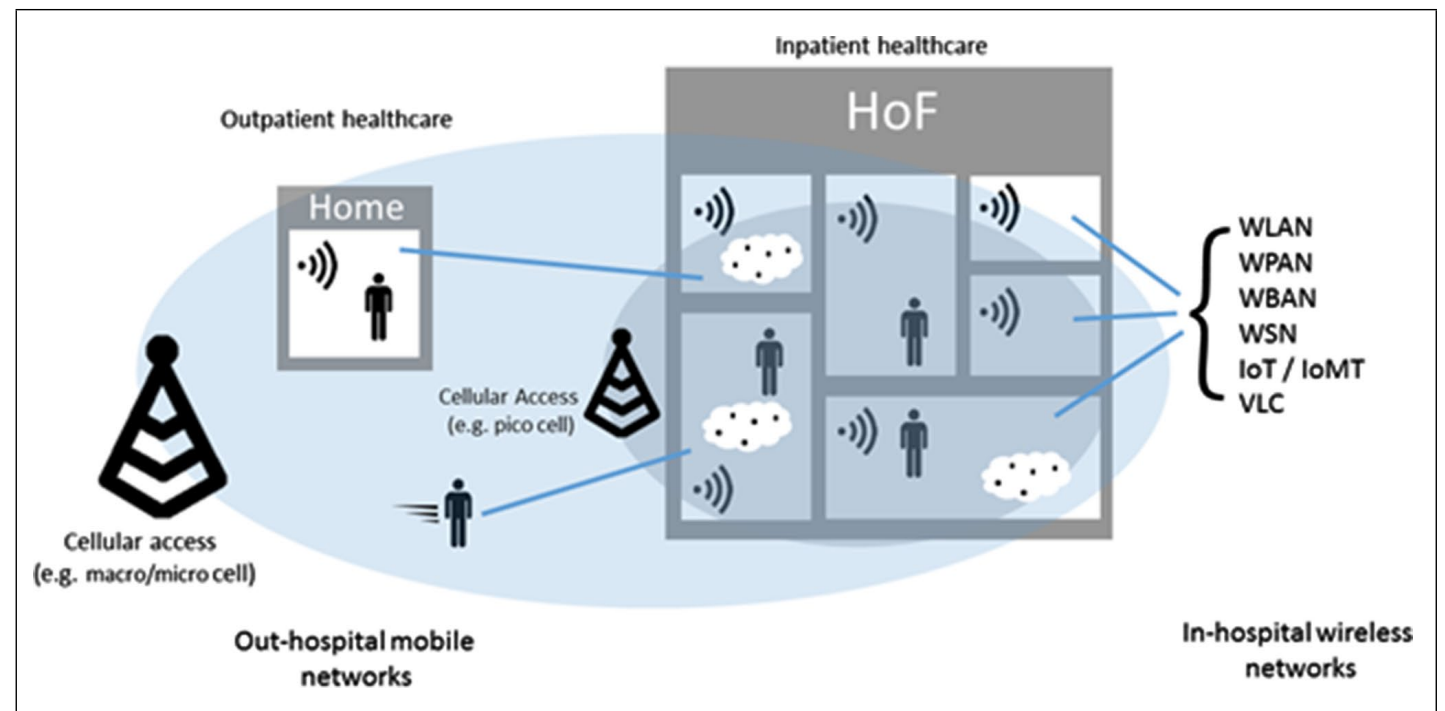

FIGURE 20: Sensor nodes collecting information about patients in hospital and home setting.

only body temperature, but also heart rate and respiration rate. The RGB camera is able to measure blood volume pulse (BVP) through the variations of light absorption on the human face [24]. Fig 21] shows a thermal image of a subject being observed.

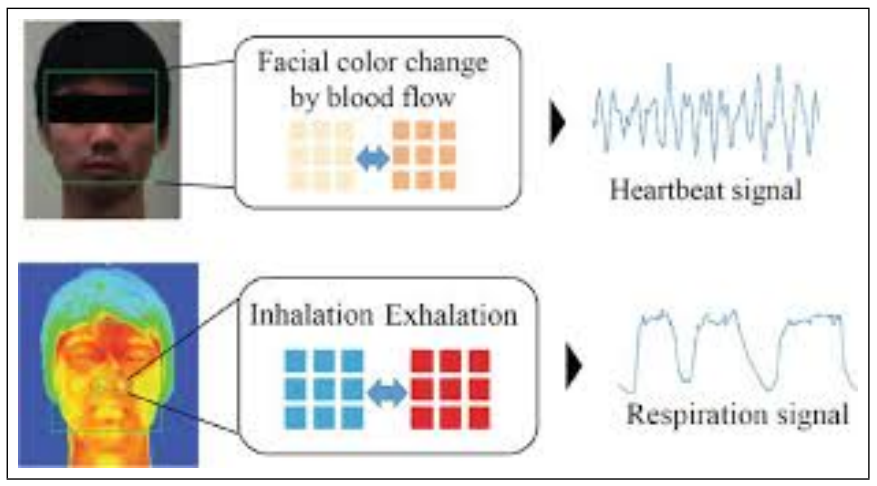

FIGURE 21: An example of a thermal image

\section{CONTACTLESS SENSORS: APPLICATIONS}

In this section, we will discuss the applications of contactless sensors in human's vital signs monitoring. We will study the latest implementation of this emerging technology using FMCW, CSI, WSN, RF, and camera based.

\section{A. FMCW-BASED}

All the approaches presented in this subsection use the FMCW technique to process and analyze the signals that are reflected off the objects, introductory details about this technique was presented in the preliminaries section.

1) Authors in [11] developed a human vital sign monitoring system based on $77 \mathrm{Ghz}$ mm-wave FMCW radar. The system, shown in Fig. 22, also utilizes compressive sensing based on orthogonal matching pursuit (CS-OMP) algorithm and rigrsure adaptive soft threshold noise reduction based on discrete wavelet transform (RA-DWT) algorithm to separate and reconstruct breathing and heartbeat signals. According to the authors, results indicate that the proposed algorithms are able to filter out noise and improve accuracy, where the accuray of respiratory rate and heartbeat rate was about $93 \%$.

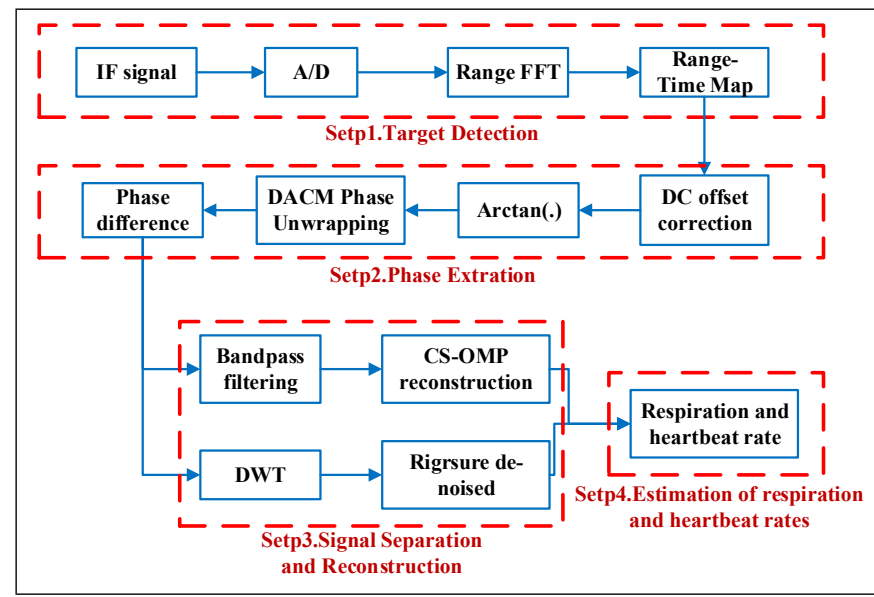

FIGURE 22: Human breathing monitoring using $77 \mathrm{GhHz}$ mm-wave FMCW radar [11].

2) Authors in [26], developed a short range-Doppler radar (1m) that may be used in many applications, like driving, health (through the monitoring of the heart rate), and security screening. They designed it according to the following scheme, see Fig. 23 It has two helical antennas, each with a 40-degree beam-width, the upper antenna is used to illuminate the heart, and the lower antenna is used for motion compensation, this system uses the ISM band at $2.4 \mathrm{GHz}$ and $2.5 \mathrm{GHz}$. 


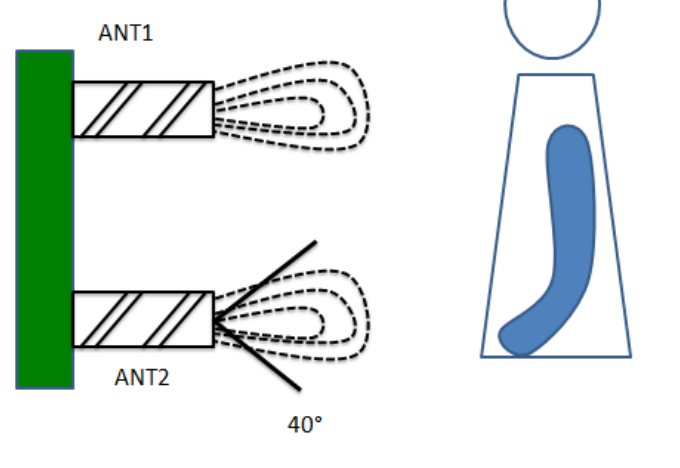

FIGURE 23: Dual antenna Doppler radar design to supervise vital signs [26].

The front view and the circuit of the proposed radar are shown by Fig. 24, where the antennas are constructed by winding copper tape on a low-loss acrylic tube.

3) A CMOS Doppler radar sensor was developed in [27], to estimate motion from heart rate and respiration rate, up to range $1 \mathrm{~m}$ and $1.5 \mathrm{~m}$ respectively, authors integrated this Doppler radar transceiver in printed circuits on a simple CMOS chip which has dimensions $5 \mathrm{~cm} \times 10 \mathrm{~cm}$, where patch antennas ASP PT 2988 are used for both transmission and reception, with a $60^{\circ}$ by $80^{\circ}$ beam-width and $8-\mathrm{dB}$ gain. The authors made two contributions, in the first, they gave the theoretical derivation as a prediction tool, and second, they achieved an experimentation validation by using PC with Matlab, as digital signal processing(DSP) for SNR.

4) Insomnia monitoring at home is essential for both diagnosis and treatment, authors in [28] proposed the EZ-Sleep system for this purpose. Moreover, the monitoring is done without any contact on the human body. Authors begin by giving the general parameters relating to insomnia and sleep, as shown in Fig. 25 In the figure, SL is the sleep latency, which means the time from coming to bed to the beginning of sleep, TST is the total time of sleep, TIB is the total time spent in the bed, and WASO (wake after sleep onset), is the total time of weakness after sleep. The EZSleep standalone is shown in Fig. 26 It consist of four processes that cooperate in gathering the subject's details to realize the application: First, capturing the user's location in the home using Frequency Modulated Carrier Waves (FMCW), and an array antenna to separate other reflected RF signal and focus only in the location, this permit to monitor the location and breathing for future use. Second, inferring bed area, it defines where the user stays stationary (chair, bed, desk, couch) then classify it as bed or no-bed, by using

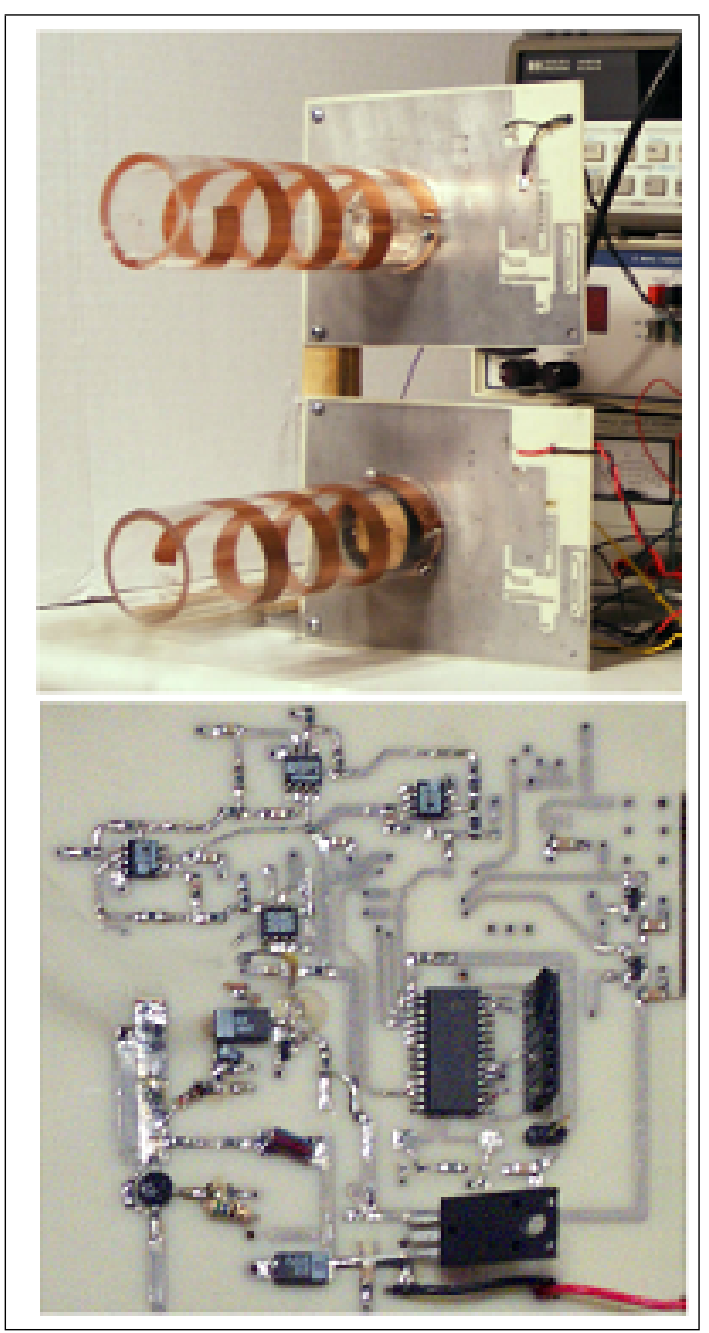

FIGURE 24: Antenna and circuit of the Doppler radar[26]

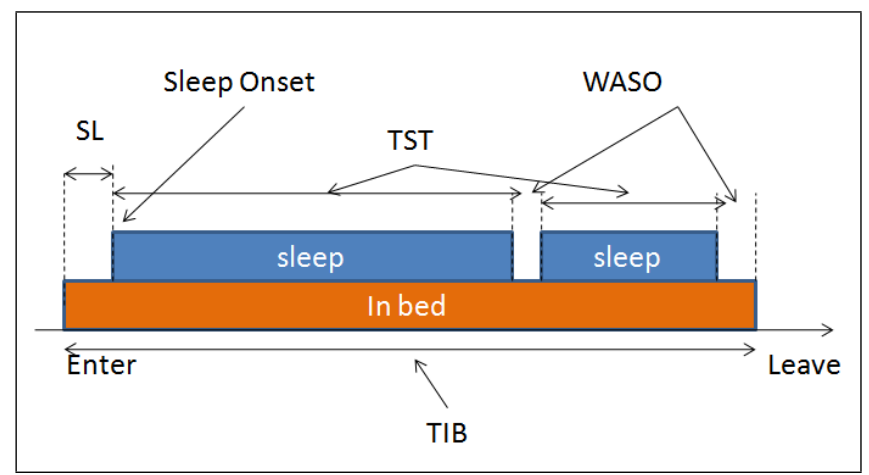

FIGURE 25: Sleep parameters [28]. 


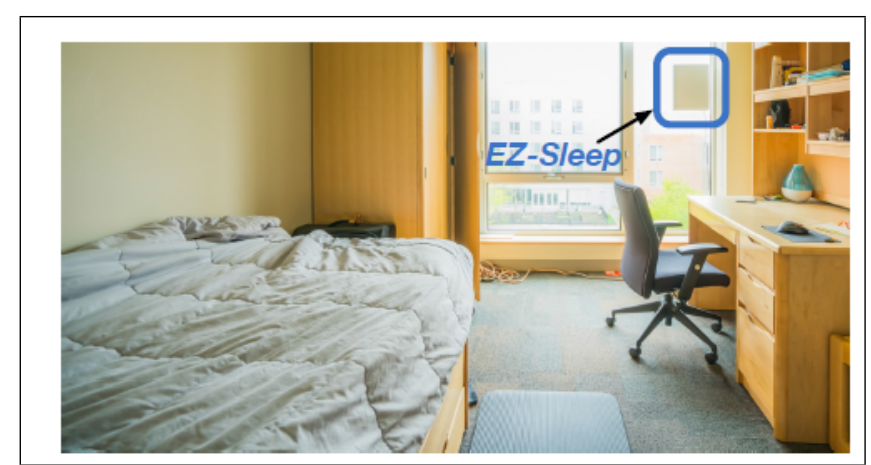

FIGURE 26: EZ-Sleep setup in one of subjects' bedroom[28]

machine learning and image processing tools. Third, detecting bed entry and exit by using Hidden Markov Model (HMM). Fourth, a neural network model is used to classify sleep versus awake.

5) Authors in [29] proposed a system to monitor and supervise breathing and heart rate signs through wireless signal and without any body contact. They also studied and presented the major challenge that must be addressed for complete efficiency in this system, that is, the inhale and the exhale effect on the reflected signal time as shown in Fig. 27 The proposed system is

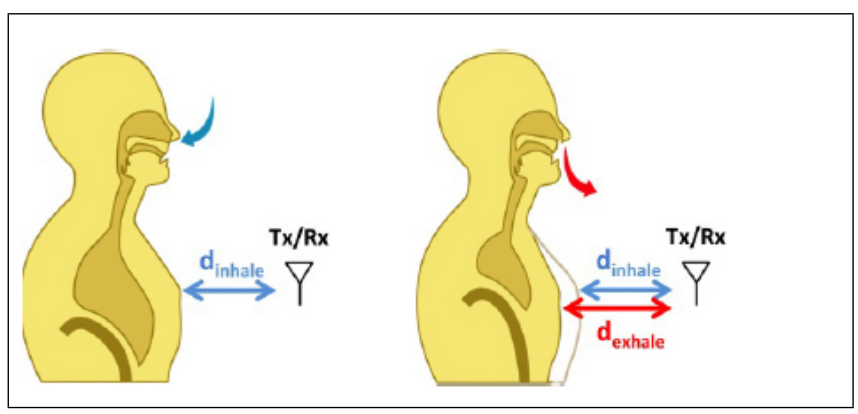

FIGURE 27: Inhale and exhale motion[29]

called Vital-Radio, and it operates according to the following three steps: First, vital-radio transmits a wireless signal, then separates the reflected signals from different objects into buckets according to their TOF's, as shown in Fig. 28 using FMCW technique. After the separation of the reflected signals into buckets, VitalRadio eliminates the signal of the static objects like furniture and walls. Second, Vital-Radio analyzes each bucket to identify those giving breathing and heart rate. Moreover in the third step, vital-Radio extracts breathing and heart rate by applying signal processing through FT. Vital-Radio differs from previous systems due to its ability to monitor several peoples simultaneously.

6) Authors in [30], began by presenting the importance of the study of behaviour in the home, for either academic goals like social research or to be used by health-care givers. They then proposed a wireless system called

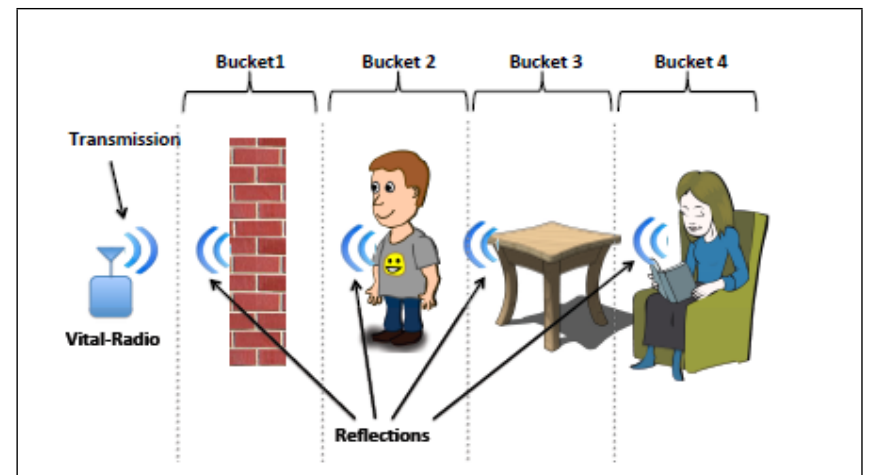

FIGURE 28: Separating reflectors into different buckets[29].

Marko, with the ability to collect information about the user's behaviour at home, without asking them to write diaries, answer questions or wear sensors. As an example of the user behaviour at home, consider when a person reaches out to the fridge, indicating the desire to eat. The proposed Marko system can be used to treat multiple users in the same home. For example, for a couple who live together Marko can answer the following questions: Does the couple sleep in the same room/bed? Who wakes up First? Who prepares dinner? Does the couple eat together? The proposed system accomplishes its task through the following steps: Marko system transmits an RF signal and processes its reflections to extract two types of data, $\mathrm{RF}$ frames and short user expected direction of motion. It uses FMCW technique as a tool for the first goal, then uses a simple technique to compute user locations. It then connects locations of the consecutive frames to construct short trajectories called tracklets. After this step, Marko system starts to eliminate extraneous data concerning the static objects, then uses a conventional neural network classifier to identify users by using RF frames and tracklets holding to the same time interval as shown in Fig. 29 .

7) A passive fall detection system (Aryokee) is proposed in [31]. It is a multi-antennas wireless system composed of three essential components as observed in Fig. 30. In the figure, fall detector, stand-up detector, and state machine take as input a short time window of RF signal transmitted by the FCMW technique. The first two component operate in parallel, each one has two cascading CNN's and a none-maximum suppression (NMS). The state machine takes as input the results of the two previous components to infer the exact fall time and fall duration.

8) Authors in [32] combined convolutional and recurrent neural networks to detect sleep stages using RF reflection. This prediction model consists of three components: feature encoder, sleep-stage predictor, and source discriminator. As shown in Fig. 31, the predictor cooperates with the encoder to predict one of 


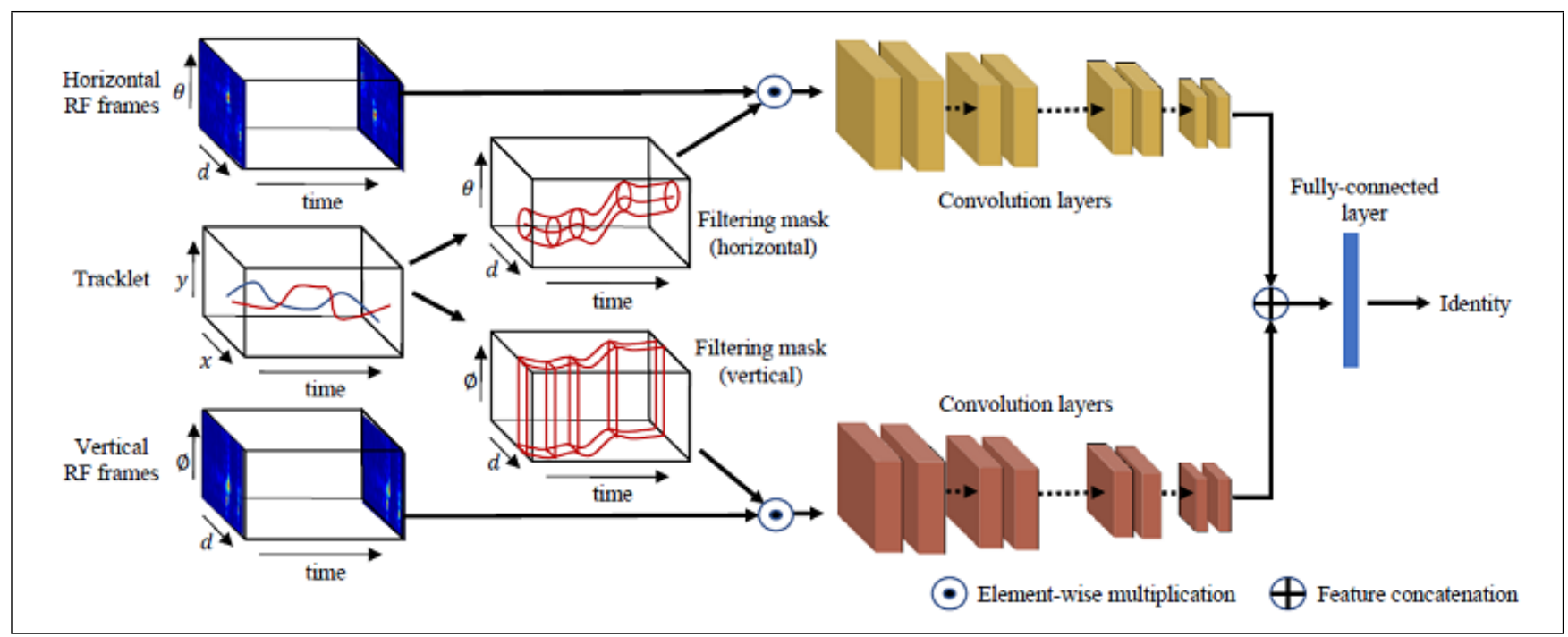

FIGURE 29: The identification CNN [30]

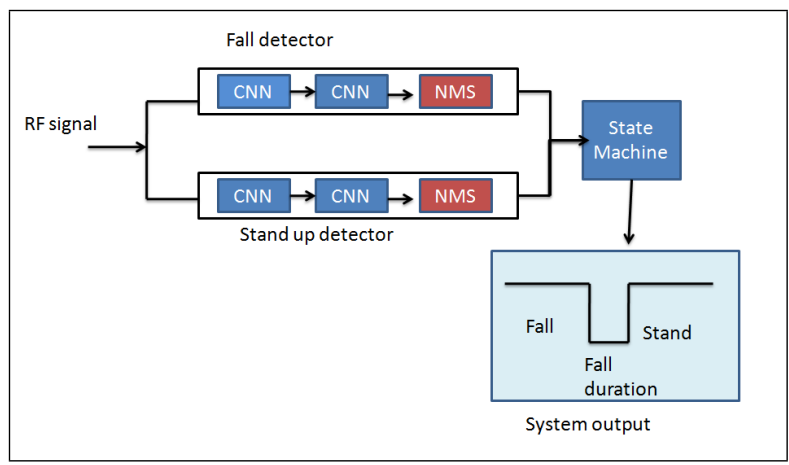

FIGURE 30: Fall monitoring system [31].

the following states (Awake, Light sleep, Deep Sleep, and REM (Rapid Eye Movement)), and prevent the discriminator from decoding the source label.

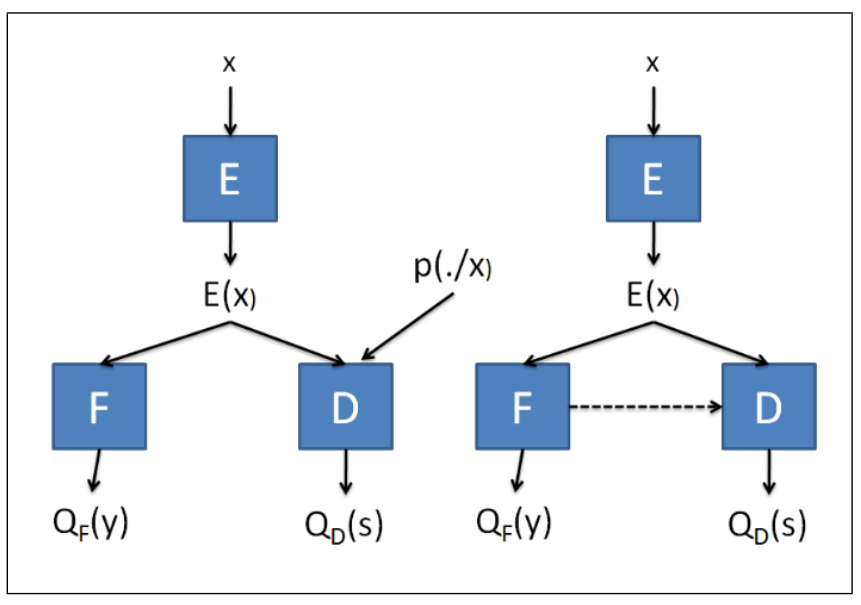

FIGURE 31: Ideal and extended game model [32].

In the figure, $\mathrm{x}$ is a 30 -second $\mathrm{RF}$ spectrogram and $\mathrm{y}$ is the predicted sleep stage.

9) Authors in [33] developed WiGait, which is a home sensor shown in Fig. 32 used to monitor gait velocity and stride length according to the following steps: First, the device takes a set of locations provided by the wireless localization system, then identifies periods when the person is stationary or moving in the place to remove them. Second the system processes the remainder to focus only on the walking periods. Third WiGait estimates the gait velocity and stride length using stable phase based on time series.

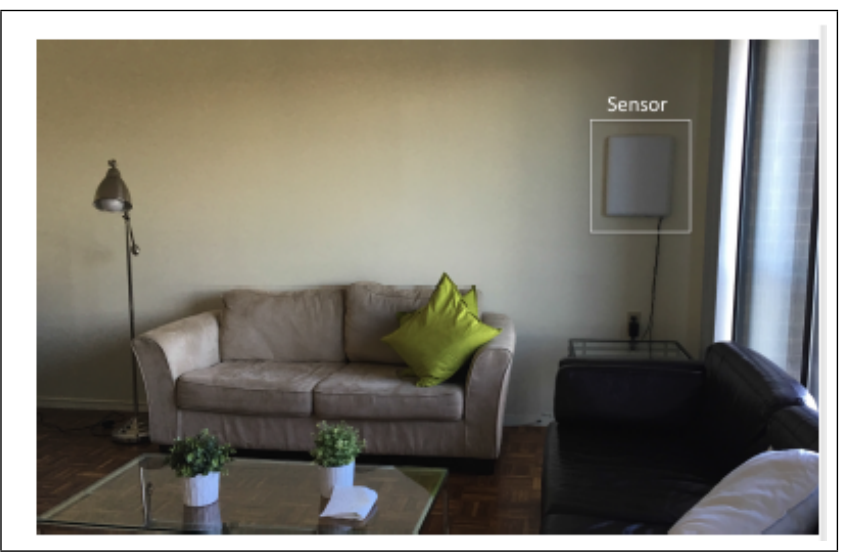

FIGURE 32: WiGait sensor system 33]

10) Author in [34] utilized FMCW-based radar system to monitor the respiratory rate and the heartbeat of a patient in a challenging indoor environment in order to simulate ambient assisted living (AAL) conditions. The authors considered four different scenarios where the patient's chest, left, right, and back side were facing the antennas. Moreover, the authors compare the performance of the proposed system with a pho- 
toplethysmograph and a respiratory belt for all the four cases and conclude that the proposed system measures both respiratory rate and the heartbeat with great precision. The designed system is a SISO FMCW-based radar working with $5.8 \mathrm{GHz}$ industrial, scientific, and medical (ISM) band shown in Fig. 33 .

11) Authors in [35] developed a wireless healthcare life and vital sign detection system using FMCW radar. Unlike conventional systems, the authors claim that the proposed system is able to detect motionless patients by analysing inherent characteristics of human respiration motion such as spatial correlation and periodicity. Moreover, multiple algorithms are employed to remove the effects of the environment and distance. Results indicate that the proposed system is able to accurately detect motionless patients. Fig. 34 shows the flow chart of life detection.

12) Authors in [36] use Emerald, which is a contact free wireless sensor utilizing machine learning for deep analysis of movement disorders. The authors use the device to measure the progression of facioscapulohumeral muscular dystrophy (FSHD). According to the authors FSHD affects around 16,000-38,000 USA citizen, moreover, its effects slowly progress over the years making it extremely difficult to track using hospital facilities. Twelve FSHD patients were continuously observed for three months using two of this device for each patient (One in the living room and one in the bedroom). The devices collected about 40,000 movement data which were processed to extract gait and time to exit the bed. The condition of the patient was raked from mild to severe and allocated a clinical severity score (CSS) from one to four. According to the authors, results indicate that there is a relationship between time to exit bed and CSS.

13) Authors in [37] also used Emerald to monitor postoperative recovery process for patients recovering from endometriosis surgery. The study was conducted on three women where the device was placed on each of their homes to study the patients movement and sleep for five weeks before surgery and five weeks after surgery. The patient was also required to conduct a daily pain assessment survey where they rate the level of pain they are experiencing. According to authors, results indicate that the longer it took the patient to fall asleep the higher the pain rating was the next day.

14) Authors in [38] used infrared images to monitor the head position of patients suffering from obstructive sleep apnea (OSA). The authors use three machine learning algorithm to process the images. Data from fifty patients with different levels of OSA severity was collected. According to the authors, the best performing algorithm Darknet19, correctly identified lateral and supine head position with an accuracy of $92 \%$ and $94 \%$ respectively.

15) Moreover, authors in [39] deployed the Emerald device which uses radio waves to detect the agitation and pacing pattern of patients suffering from Alzheimer's disease (AD). The authors claim that the device is able to detect changes in movement patterns that are able to reveal underlying behavioral symptoms and environmental triggers. The device was installed for seventy days in the house of a single patient suffering from AD. According to the authors results indicate that there is a relationship between pacing of the patient and environmental triggers.

16) Authors in [40] exploited the RF signal to view the human skeleton without wearing any smart device. The essential elements in this process are RF signal sent by a radio device, the antenna array is used to define the angle direction of the arriving signal. FMCW is used to measure RF reflections and static reflection elimination and focus only on the reflection of the human body. These elements are integrated into the developed RFcapture device. The big challenge facing the proposed system is: there is a lot of RF signal reflected away from the device, and only a subset reflected towards it as seen in Fig. 35. Authors tried to address this challenge by exploited body motion to trace the body of the person.

17) Authors in [41] proposed BodyCompass (see Fig. 36], an RF-based sleep poster monitoring device to help patients avoid bedsores after surgery, reduce apnea event, monitor the progression of Parkinson's disease, and even alert epilepsy patients to a potential lifethreatening sleeping position. The device works by analyzing RF signals that bounce off the patient using machine learning algorithms. According to the authors, the device was used to evaluate data from twentysix patients over a period of two hundred nights. The observed accuracy of the system was over $80 \%$.

18) Authors in [42] proposed the use of an RF-based home monitoring device to servile patients with Parkinson's decease (PD) focusing on gait, home activity, and time in bed. The study was conducted on seven patients over a period of eight weeks where the RF-based device was installed in their rooms. According to the authors, promising results were obtained to help better understand PD, moreover, the device can be used to treat other chronic diseases.

19) Authors in [43] claimed that RF signals may be used for inferring emotions (happy, sad, confused, etc.), then they demonstrated that the reflected RF's have information that can be used to extract breathing and average heart rate without any contact, they also presented the challenges facing this technique. First, the reflected $\mathrm{RF}$ signal is modulated by breathing and heartbeats. Second, the heartbeat in the RF signal lacks the sharp pick that characterizes the ECG signal. As a result, the boundaries of identification become hard, to address these challenges, the authors designed and developed the EQ-Radio system, which consists of three compo- 


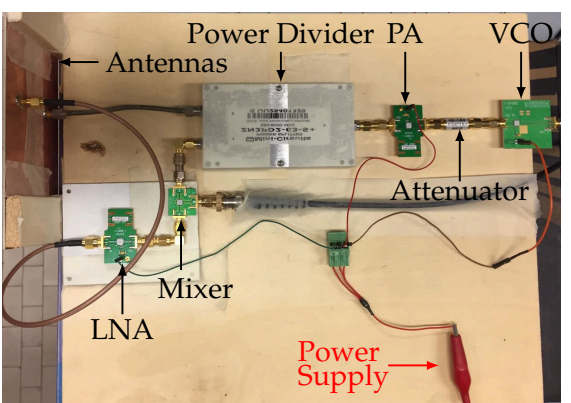

(a)

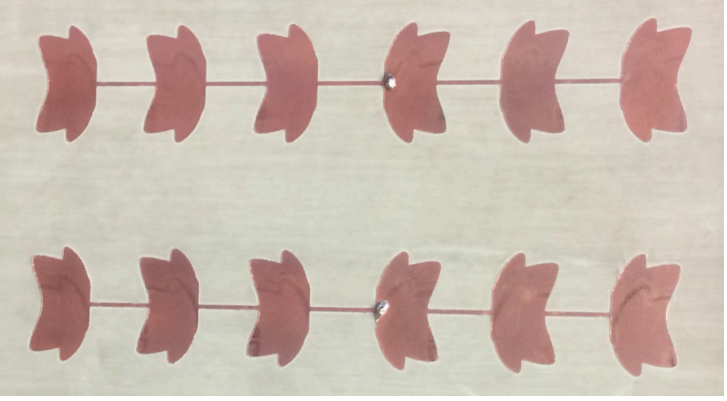

(b)

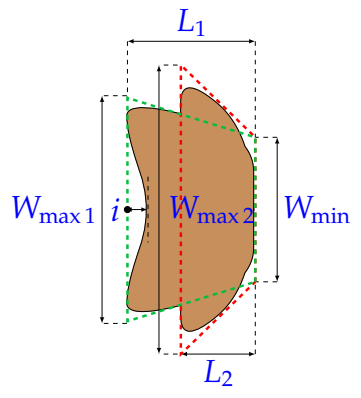

(c)

FIGURE 33: (a) Radar system, (b) antennas, and (c) path orientation [34

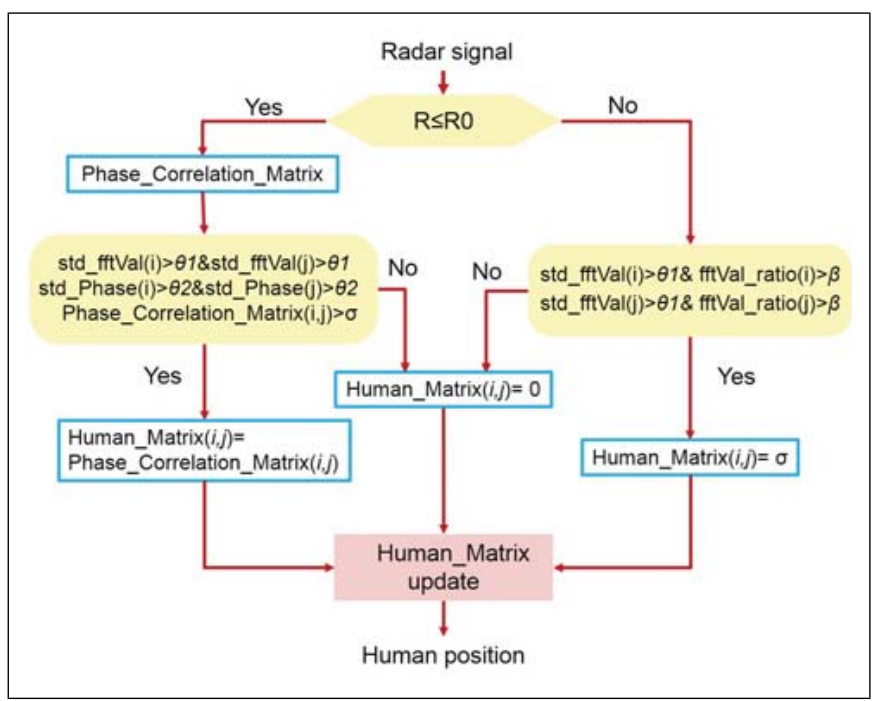

FIGURE 34: Flow chart of life detection [35]

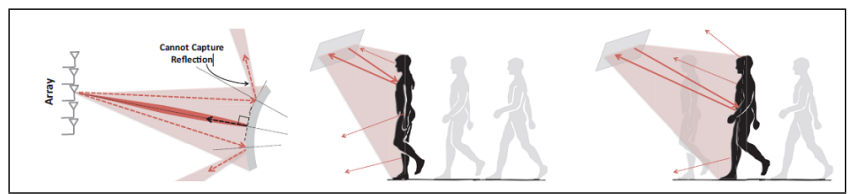

FIGURE 35: Reflections off human body [40]

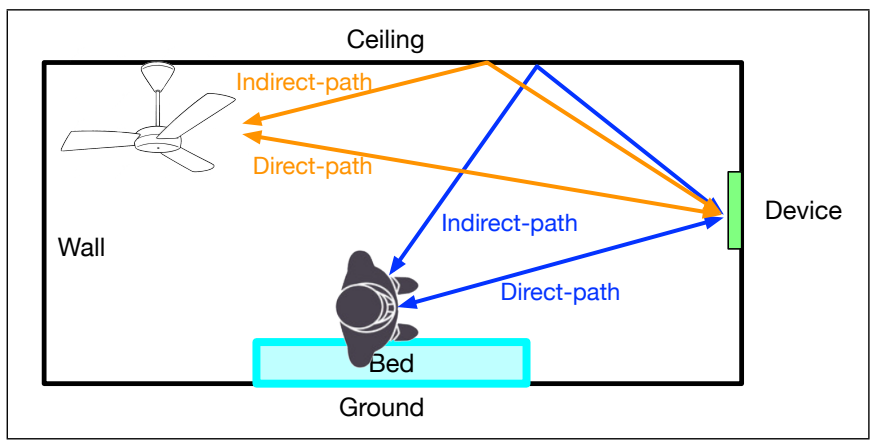

FIGURE 36: Signal reflections in a patient's room [41] nents as shown in Fig. 37 First, the radio transmits

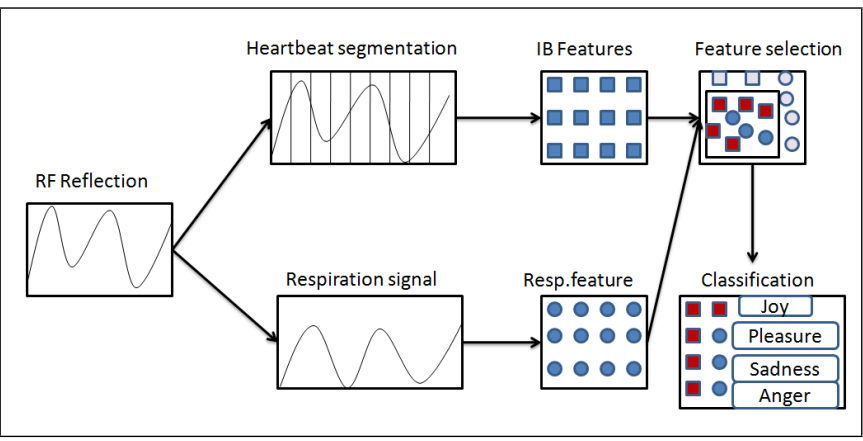

FIGURE 37: EQ-Radio Architecture [43].

a low power signal, then estimates its reflection time using the FMCW technique previously described, this is used to differentiate between reflected signal off different objects. Each one in a specific bucket, EQRadio achieves a pre-processing to mitigate noise and to improve the signal-to-interference-and-noise ratio (SINR). Second, the heartbeat extraction algorithm takes the reflected signal after the pre-processing phase previously achieved as input and returns a series of segments as output. This output is used by the third component which is the emotion classification, to determine an emotion according to the received physiological signals using an SVM classifier. Through experimentation, authors showed that EQ-Radio performs better compared to ECG-based and image-based systems.

20) Authors in [44] proposed Witrack system, for tracking users in two scenarios, these are line of sight and nonline of sight (through a wall). The system is composed of four antennas in $\mathrm{T}$ form. One for transmission and three for reception as can it be observed from Fig. 38. The device transmits an RF signal then computes and estimates the TOF using FMCW technique, it then focuses on the human's body reflections by suppressing those of the static objects after de-noising. Witrack 


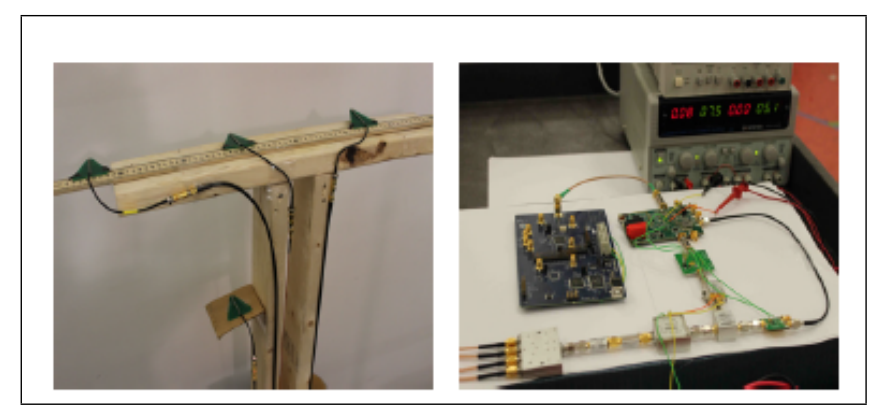

FIGURE 38: Antenna 'T' and FMCW signal generator[44]

estimates the traveled distance from the transmission antenna to the human and back to the reception antenna, in other words, three estimated distances are used to identify the user's location. In the end, Witrack uses elevation through the Z-axis to detect fall with the following two conditions. First, the elevation change must be significantly observed. Second, the final value of this elevation must be near to the ground level.

21) Authors in [45] proposed a wireless system called WiTrack2.0, as an improvement to WiTrack [44], it has 5 antennas for transmission and 5 antennas for reception as shown in Fig. 39. Unlike Witrack, it can localize multiple users in a multi-path indoor environment by transmitting an RF signal and then processing its reflection. It has two essential components, the first component is the FMCW to deal with the multi-path effect, and the second component is the successive silhouette cancellation algorithm to overcome the nearfar problem.

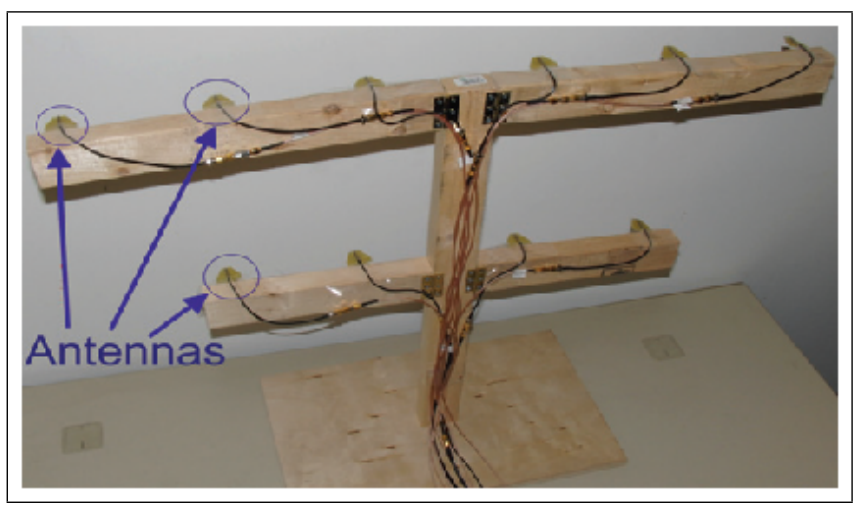

FIGURE 39: WiTrack2.0 antenna system 45

\section{B. CSI-BASED}

Channel state information (CSI) contains a plethora of hidden information about the propagation environment, hence, it can play an important role in gathering and extracting unknown information that extends the human sense about the environment. In this section, we present several approaches and contributions of CSI-based technique in the contactless technology used to supervise user's vital signs.
1) Authors in [17] proposed a WiFi CSI-based human activity detection for assisted-living patients. The system works by analysing the Doppler shifts in the WiFi CSI caused by human activity. Three case studies are performed in the experiment, through-wall detection of vital signs, daily activity recognition in residential home, and activity monitoring in a residential environment, sequentially inference as case 1 , case 2 , and case 3 respectively. Fig. 40 shows an example of 24 -hour monitoring in a house environment using the proposed system.

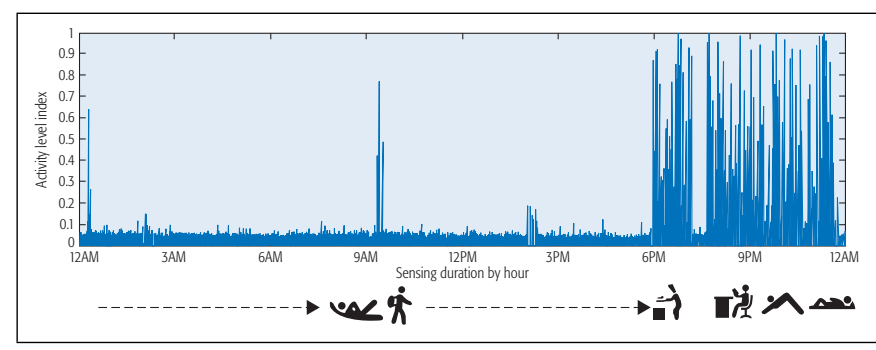

FIGURE 40: 24 hour monitoring using WiFi CSI [17]

2) Authors in [46] proposed a non-invasive method for monitoring the respiration rate using received signal strength measurements (RSS). The goal of the proposed work is to address two critical problems encountered when observing breathing using a single transmitter-receiver pair. The first problem is the difficulty in observing the breathing signal using RSS because inhaling and exhaling causes finite change in propagation channel. The authors addressed this problem by increasing the signal-to-noise ratio (SNR). The second problem addressed is the distinction of other movements of the person from breathing motion, these movements dominate most of the observed RSS response. To counter this problem, authors suggest to identify time instances of the movements as monitoring continues, then the device is disabled at these instances in the long run. The following scheme presents the different components of the breathing monitoring system as observed in Fig. 41. First, the noised RSS measurement is pre-filtered to increase the SNR then downsampling is performed. Second, the mean removal is applied to the sampled signal, then passed through a motion interference detector and finally through a breathing estimator.

3) Motion detection become an essential component in most modern systems, therefore, authors in [47] proposed WiDetect system. Unlike most existing motion detectors which require particular installation, calibration, and have limited coverage, WiDetect is a highly accurate, calibration-free, and low-complexity wireless motion detector. The proposed system exploits the statistical properties of electromagnetic (EM) waves to find a relationship between the autocorrelation function 


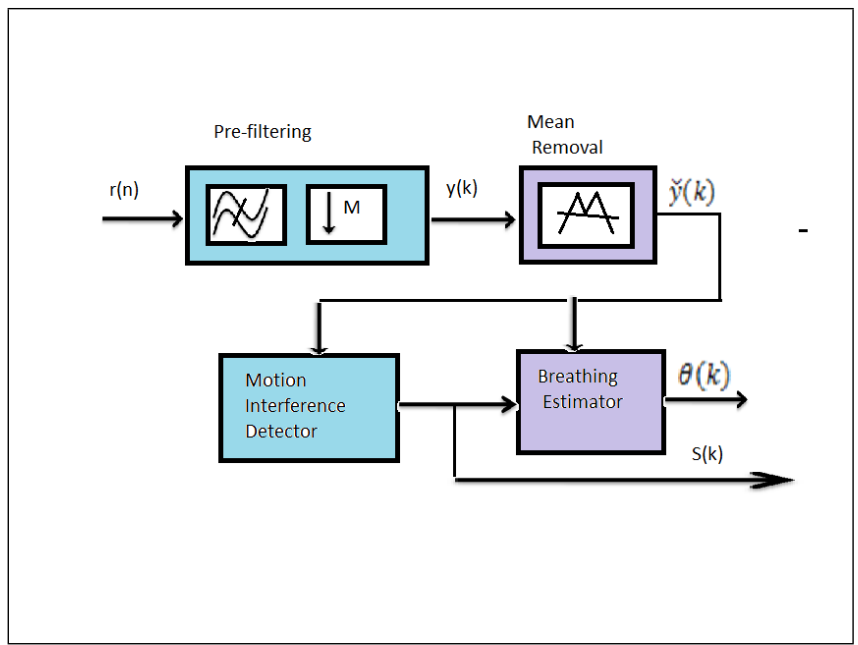

FIGURE 41: Breathing monitoring system components [46].

of the physical layer CSI and movement in the surrounding environment. Authors consider buildings and rooms with scatters that diffuse EM as reverberation cavities. This is because the building or room contain internal multipath signals between a transmitter $(\mathrm{Tx})$ and a receiver $(\mathrm{Rx})$ as shown in Fig. 42 .

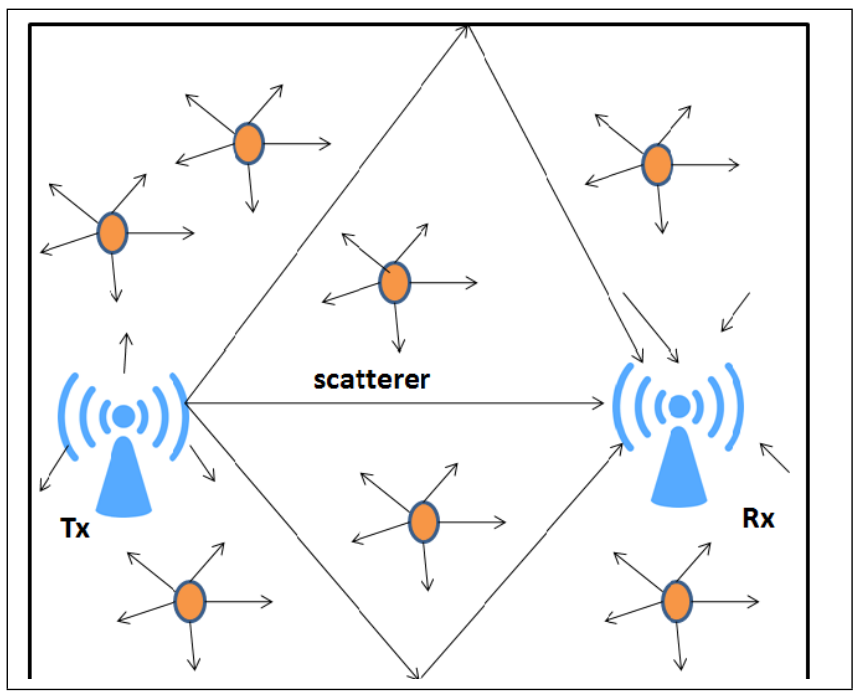

FIGURE 42: Propagation of radio signals in scattering environment [47].

4) Authors in [48] used reflected Wi-Fi signals to recognize humans through their gait patterns in an indoor environment utilizing the architecture depicted in Fig. 43

They used two transmitter antennas (such as a Wi-Fi router) and a receiver antenna (such as a laptop). The proposed system operates according to the following steps:

The system starts by collecting the CSI measurements, then extracts the principal component from CSI mea-

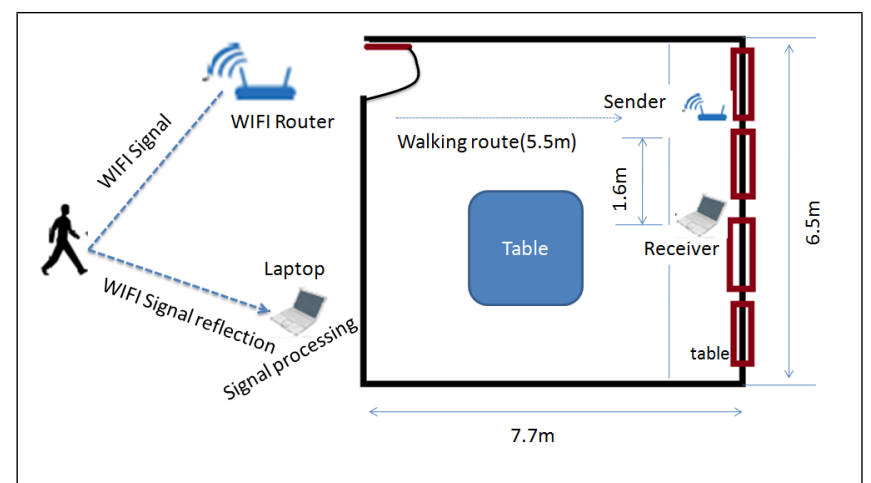

FIGURE 43: Application scenario and data collection environment [48].

surements using the Principal Component Analysis (PCA) technique. Next, it transforms the resulting PCA into a spectrum using short time fourier transform (STFT), then enhances this spectrum by applying frequency domain denoising algorithms to reduce the noise. After that, a machine learning technique (SVM classifier) is applied to classify the extracted feature into two classes (the person understudy and others).

5) Authors in [49] designed WIFID system, which is a device-free system used to identify humans indoors. The system exploits the ability of PHY layer CSI to obtain the frequency assortment of a widband channel. The layout of the experiment is shown in Fig. 44 A pair of Wi-Fi transmitter and receiver is deployed. The transmitter has a single antenna while the receiver has three antennas. Moreover, the transmitter continuously broadcast a signal every 3 milliseconds. After receiving this signals the system runs the following three modules:

First, WIFID analyzes CSI to focus on the user segment and to extract a novel feature of sub-carrier amplitude frequency (SAF). Second, WIFID applies PCA on the resulting SAF, and third, WIFID uses an SVM classifier for classification.

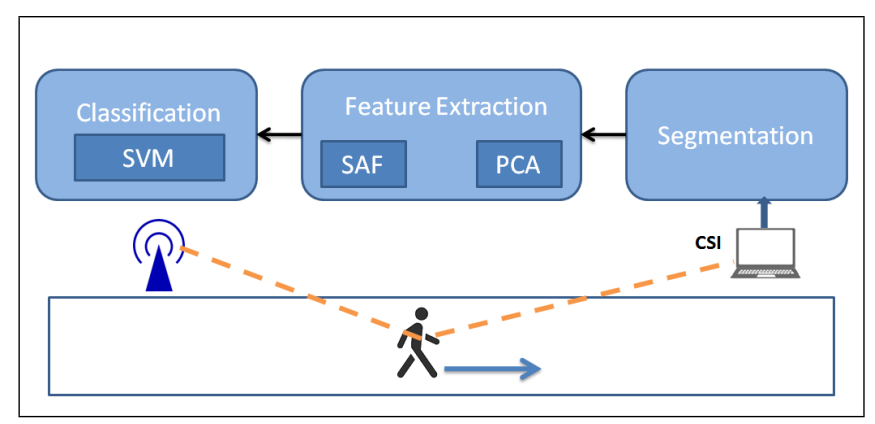

FIGURE 44: WFID architecture [49].

6) A non-contact system to detect paraparesis is proposed by authors in [50]. The proposed system has SIMO architecture and operates on a pipeline mode according 
to the following steps as observed in Fig. 45. First, the system collects data through wireless sensing. Second, the collected data is passed to the pre-processing stage where the system uses a Humpl filter to detect outliers values, then applies Wavelets for de-noising signal and finally uses data calibration to increase sample data. Third, a CNN classifier is applied to classify data and detect paraparesis.

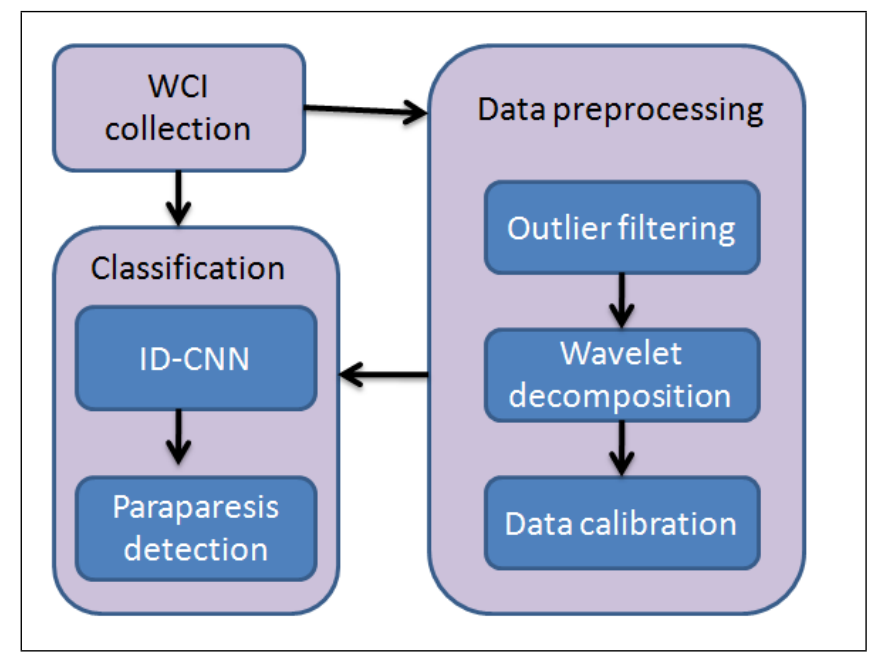

FIGURE 45: Workflow of the 1D-CNN system [50].

7) Authors in [51] used CSI from WiFi 802.11n in a home environment as shown in Fig. 46, to detect human activity such as sitting, standing, walking, and running. Moreover, the human activity recognition system is able to detect the number of people in a room as well as recognising human fall, which is very instrumental in the case of assisted living conditions. The proposed system is developed using two algorithms: support vector machine (SVM) used for classification and long short-term memory (LSTM) recurrent neural network.

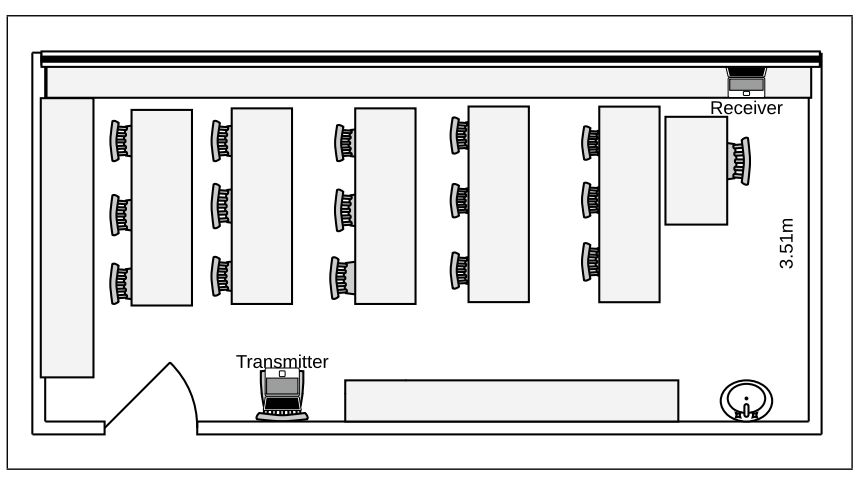

FIGURE 46: Human activity recognition room setup [51]

8) Author in [52] proposed a reconfigurable intelligent surface (RSI) based radio frequency system for human poster recognition. As shown in Fig. 47, the system comprises of a single transmitter, a receiver and an RIS. The RSI can reflect and modify the incident signal from the transmitter. During sensing, the transmitter continuously generate a single-tone signal of a given frequency which is reflected by the RIS and the human body before reception by the receiver. According to the authors, the proposed system is able to customize the environment to provide the needed propagation properties and a plethora of transmission channels.

9) Authors in [53] proposed a monitoring system to supervise events in an indoor environment. The proposed work exploits the information embedded in the channel(CSI) and uses commercial WIFI devices for real time monitoring. The system is made up of a feature extracting algorithm utilized during training to recognize the most distinctive sequence of CSI. The system also uses principle component analysis (PCA) to reduce noise and to remove correlation between subcarriers and links. Authors also note some challenges associated with real-time monitoring. The challenges include unknown start and end time of an event, change in occurrence instances, and precise detection with low latency. Authors propose a modified k-nearestneighbor $(\mathrm{kNN})$ classifier to address the mentioned constraints. Another problem facing real-time monitoring is durable robustness due to the eventual and unpredictable environmental change. Authors integrated in their model an unsupervised retraining algorithm that guarantees high accuracy during an environmental change. The proposed system is depicted in Fig. 48

10) Authors in [54] proposed MultiSense human respiration sensing device. According to the authors, the WiFi-based device accurately measures similar breathing pattern of multiple people in a room even when they are very close to each other. The device utilizes multiple antennas to receive a blended reflected signal from monitored individuals. Authors claim that the device is able to accurately measure respiration even in a room of four people with the mean absolute respiration rate error of $0.73 \mathrm{bpm}$ (breaths per minute).

11) Authors in [55] developed a continues real-time heartbeat monitoring device utilizing $24 \mathrm{GHz}$ continuouswave Doppler radar as well as artificial neural networks (ANNs). The device was designed to have very low latency of less than one second. Moreover, the device was tested on twelve volunteers and the results were evaluated against results from an electrocardiogram (ECG). Authors claim that results indicate that the device is viable to detect accurate heartbeat from patients. Fig 49 shows the proposed model.

12) Authors in [56] proposed WiSee, a whole-home gesture recognition system that requires neither sensors on humans nor a camera system. The system can recognize gestures behind obstacles because it uses Wifisignal that propagate not only by line-of-sight but also through the wall. WiSee achieves the recognition task by following the steps below:

First, the system extracts Doppler shifts that appear 


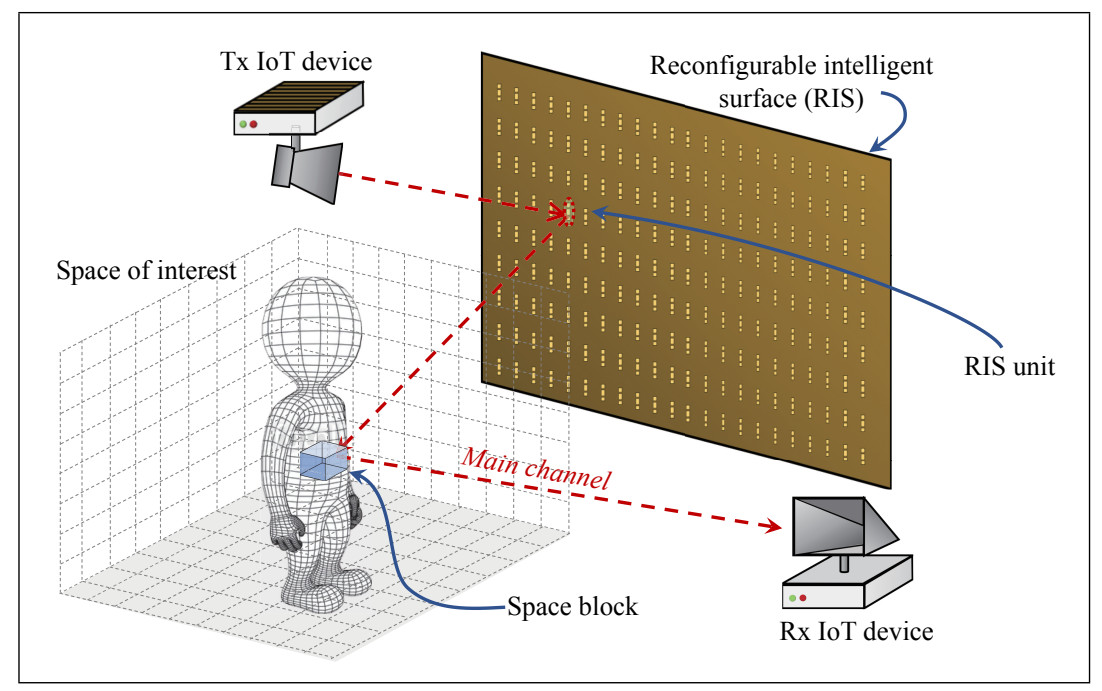

FIGURE 47: Reconfigurable intelligent surface (RSI) based radio frequency system model for human posture recognition. [52]

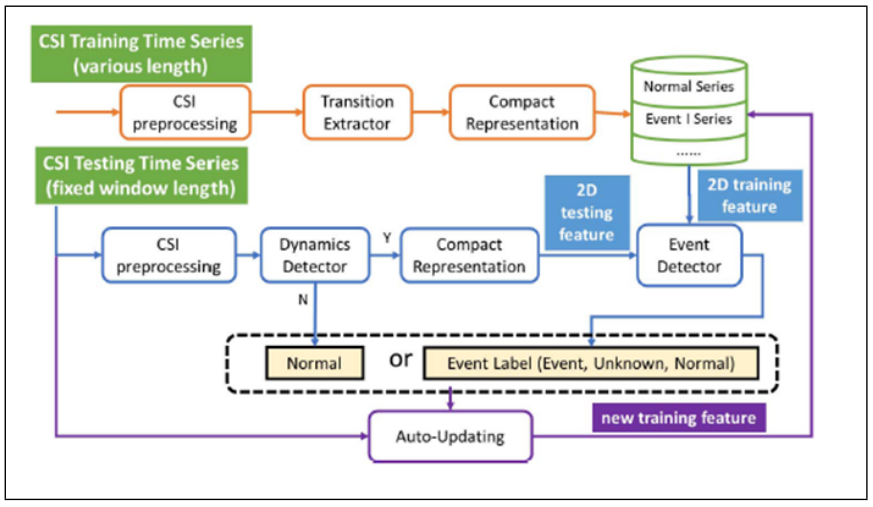

FIGURE 48: Indoor event monitoring system diagram. [53]

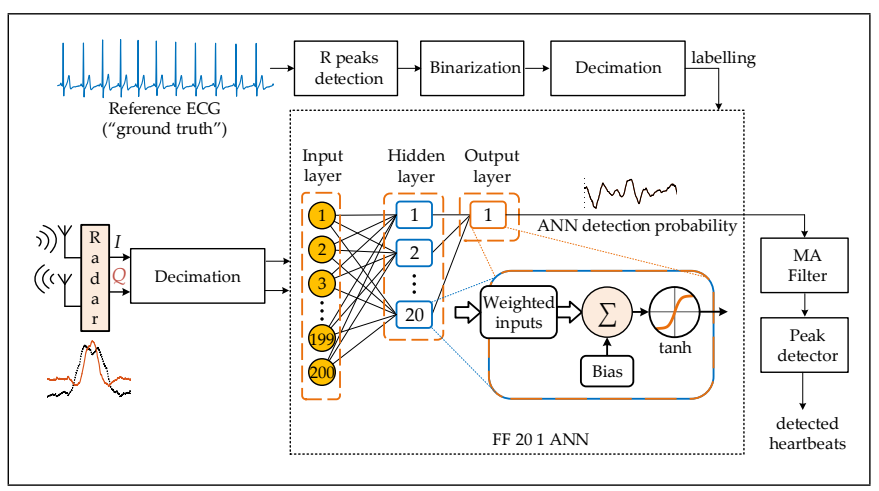

FIGURE 49: Proposed heartbeat detector flowchart. [55]

in the wireless signal, caused by the movement of the object. Second, WiSee maps this Doppler shifts to the gestures. WiSee can classify the nine wholebody gestures shown in Fig. 50. Moreover, WiSee system also takes into consideration multiple humans scenarios using MIMO.

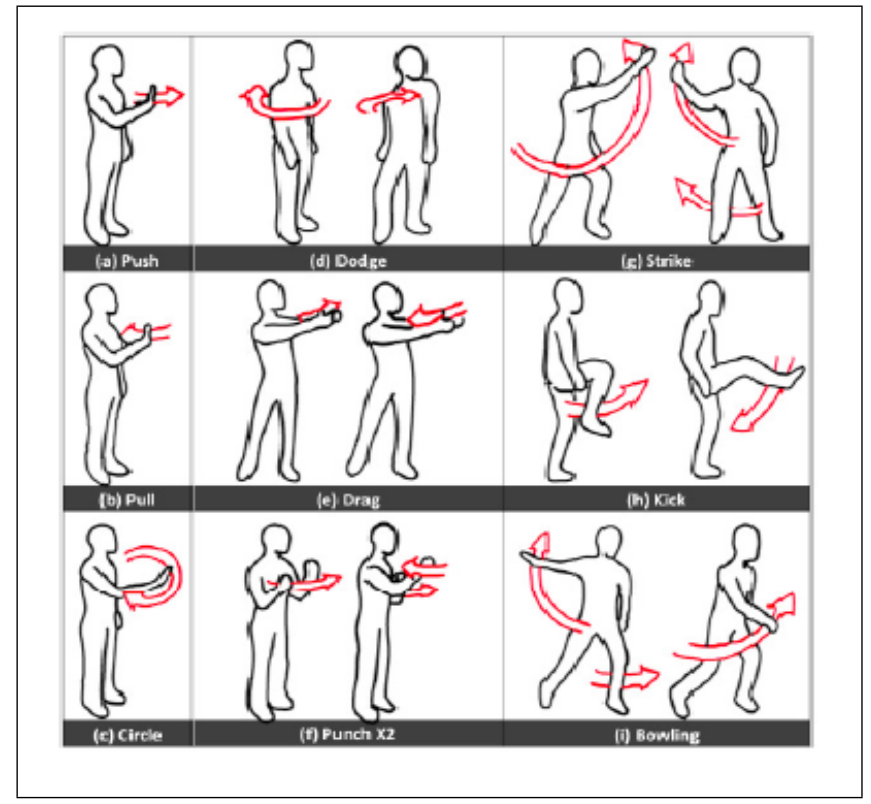

FIGURE 50: WiGait sensor system [56]

\section{WSN-BASED}

A wireless sensor network (WSN) is a set of interconnected sensors deployed in a given area to supervise a particular phenomena. The sensors mostly use multi-hop communication technique to send their data. In this section, we present three main approaches of the contactless vital-signs supervising based on a set of WSN devices.

1) Authors in [57] showed that $4 \%$ of the male and $2 \%$ of the female adult population is affected by sleep apnoea. Therefore, the authors developed the SleepMinder, which is a novel contactless technology to supervise sleep and breathing at home. The sensor operates in a license-free band of $5.8 \mathrm{GHz}$, and its emitting average 
power is less than $1 \mathrm{mV}$ and it is able to measure movement and breathing across the distance of between 0.3 and $1.5 \mathrm{~m}$. Also, it can process the case of two peoples in the bed, by combing sophisticated sensor design and signal processing to focus only on the respiration of the nearest person. The study aims to validate Sleep Minder as a sensor contactless technology with the ability to accurately estimate the Apnoea-Hypopnoea index (AHI). The model of the proposed Sleep Minder system is shown in Fig. 51 .

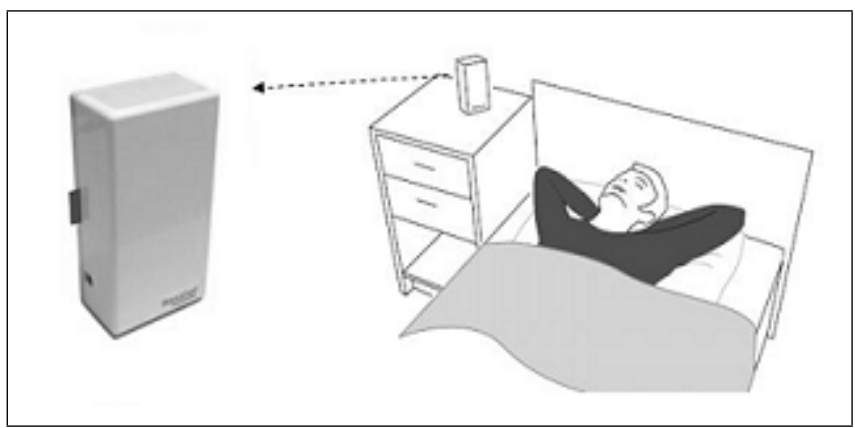

FIGURE 51: An example set up of SleepMinder in the home environment [57]

In the proposed model, algorithms used in [58] is integrated and used to analyse recordings of 129 subjects with suspected sleep-disordered breathing (SDB). Numerous observations were made such as sleep state and motion flags which were used in further analysis. Moreover, a new algorithm was used to detect SDB using phase demodulation and amplitude as well as correlation-based signal processing methods. The AHI is estimated by dividing the total number of the detected events over the Total Sleep Time (TST) as seen in Fig. 52

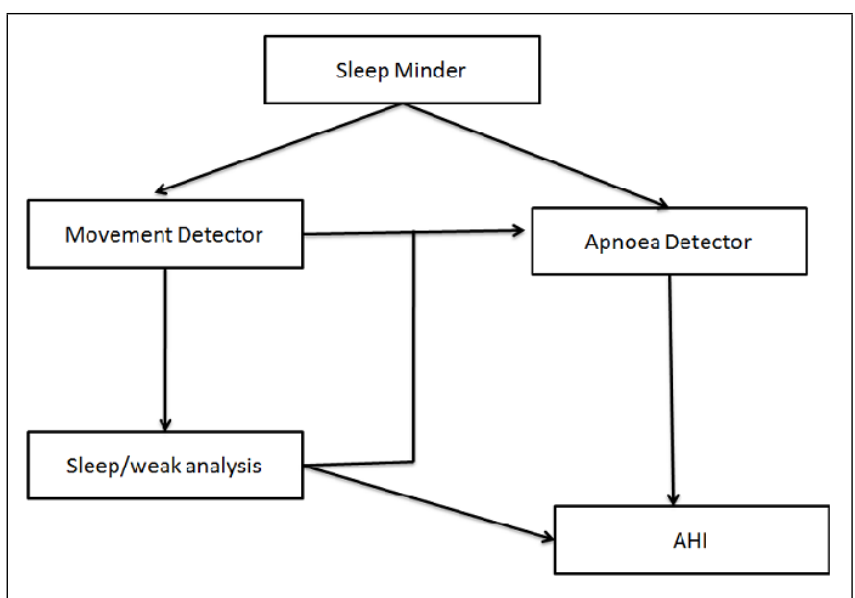

FIGURE 52: Signal processing steps [57].

2) Authors in [59] developed a WSN-based network to monitor patients' psychological parameters and track patients inside a hospital. According to the authors, the experiment was done in collaboration with Henri Mondor University Hospital Center in France. Fig. 53 depicts the setup of the experiment where the application used in the proposed system simply collects temperature and luminosity values captured by sensor.

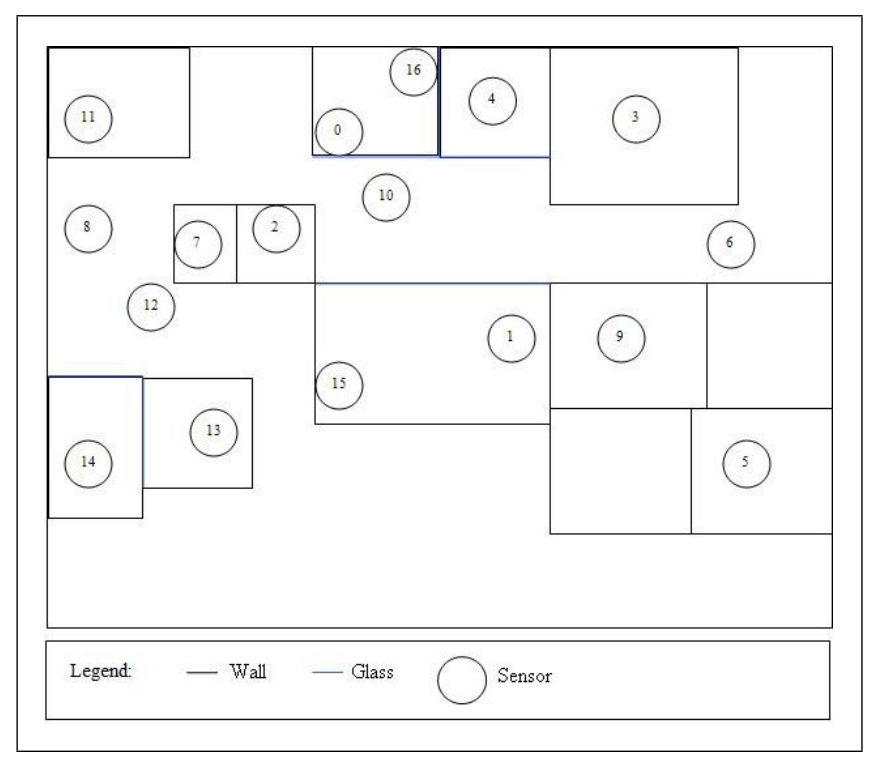

FIGURE 53: Sensor deployment in the hospital [59]

3) Authors in [60] deployed a set of sensor devices as shown by the testbed network in Fig. 54, these wireless devices (black points) collaborate with each other to localize a breathing person and estimate his breathing rate in-home using RSS. The authors demonstrated two situations. First, the case when the person is stationary (sitting, lying down, standing, or sleeping.), in this case, a prior calibration is needed. The Second situation is when a person is in motion (moving), in this case, we do not need calibration. They presented two methods to estimate the breathing rate. The primary method takes the maximum frequency at the sum, it is based on the power spectral density (PSD) calculated over links. Moreover, authors mentioned the challenge facing the RSS-based breathing rate estimation. Interference due to links sharing with other objects in the environment is one of them. To address this problem, authors use time index to identify at which time the sudden RSS change occurs. This technique is called a break point. As the second method for the localization, authors exploit the previous two techniques and the amplitude of the signal component at each link to construct the map and identify the chest position. For experimentation, they deployed TI CC2531 dongle nodes.

\section{CAMERA-BASED}

Observing the human face and body without any contact and measuring physiological signs using a camera is a technology 


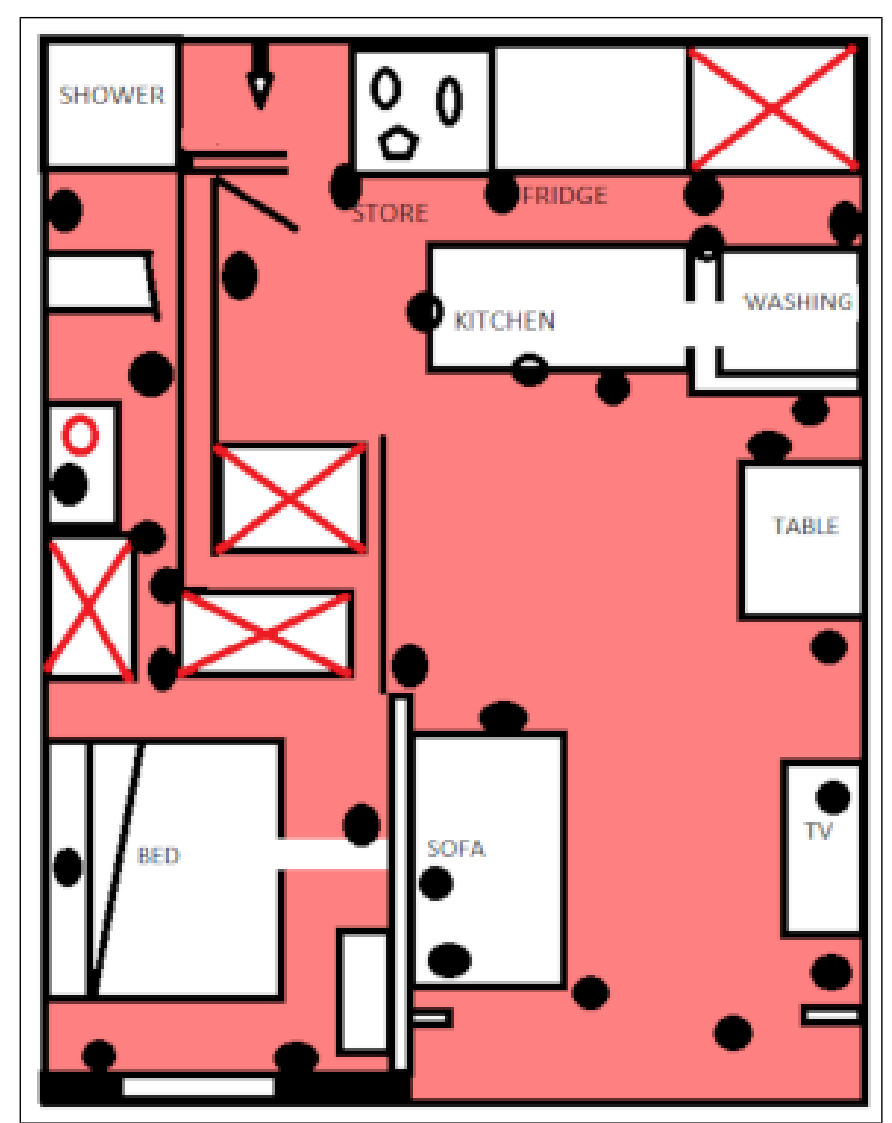

FIGURE 54: Sensor devices test-bed network [60].

that has gained research momentum in the last decade. Without the need of contact, remote cameras can be used to measure vital signs such as heart rate irregularity, breathing rate, blood oxygenation saturation, pulse transit time and body fever. According to [61] the most popular type of external sensors are cameras. In this section, some applications of camera-based sensing are highlighted.

1) A contactless vital signs measurement paradigm using RGB-Thermal image sensors is proposed in [24]. The RGB camera sensors measures blood volume pulse (BVP) using light absorption deviations on the human face. Moreover, infrared thermography (IRT) was used to measure changes in temperature near the nose or mouth during breathing. The authors conclude that the results obtained by the proposed contactless system for measurements such as heart rate, respiration and temperature are highly similar to the results obtained using contact systems such as a respiration belt and a thermometer. Fig. 55 shows the proposed contactless system.

2) Authors in [63] developed a screening system utilising thermography and other wireless sensors to observe multiple vital signs from a subject. Moreover, the authors employ machine learning and apply six different classification algorithms and compare their performance. According to authors, after performing a test where 92 people were screened using the proposed method, results indicate that there is 50\% more accuracy increment compared to using thermography alone. Fig 56 shows the proposed system model. Other previous works using infrared cameras include mass blind fever inspection [64][65]|66], infrared thermometers [67], and RGB-thermal screening with facial tracking [68].

3) Authors in [69] proposed a see-through-wall imaging radar system that is used by law enforcement agencies to observe objects behind a wall. The system is an Ultra-wideband high-resolution short pulse imaging radar paradigm operating at $10 \mathrm{GHz}$. According to the authors, the model investigates two design properties, first is the the electromagnetic wave propagation property through wall, and second is the pulse fidelity.

4) Authors in [70, 71] discussed the principal behind non-contact measuring of respiration and heartbeat using infrared/RGB facial-image. Moreover, potential applications such as the detection and observation of a person with infectious diseases were also discussed. According to the authors, an RGB camera was used to observe the heartbeat of the patient from the face due to the degree of skin exposure and ease of detection. The camera measures the reflected light from the arteries to get the heartbeat signal. Moreover, an infrared camera was used to measure respiration activity by observing the nasal-region of the subject. An infrared camera is used because during inhalation the cool air from the environment lowers the temperature around the nasal cavity and during exhalation the hot air from the lungs raises the temperature around the nasal cavity. Fig. 57 shows the principal utilized to measure respiratory and heart rate using infrared and RGB imaging as well as an infectious decease screening system.

5) The current omnipresent property of smart phones with optical sensors have provided an opportunity for lowcost vital signs remote monitoring. Authors in [72] proposed a novel system for monitoring heart rate, perfusion index and oxygen saturation of a person using a camera system from a smart phone. According to the authors, they employed principal component analysis (PCA) technique and the results obtained demonstrated that the proposed system performs better than conventional systems. Fig. 58 shows the proposed system model, where, HR, PI, and $\mathrm{SpO} 2$ represents heart rate, perfusion Index, and saturation of peripheral oxygen respectively .

\section{NEW HARDWARE TECH FOR WIRELESS SENSING}

In this section, we will discuss new advancements in hardware platforms that facilitate research on wireless sensing. Numerous hardware platforms that facilitate research on wireless sensing have emerged due to its resent popularity among researchers and the high potential of contactless sens- 


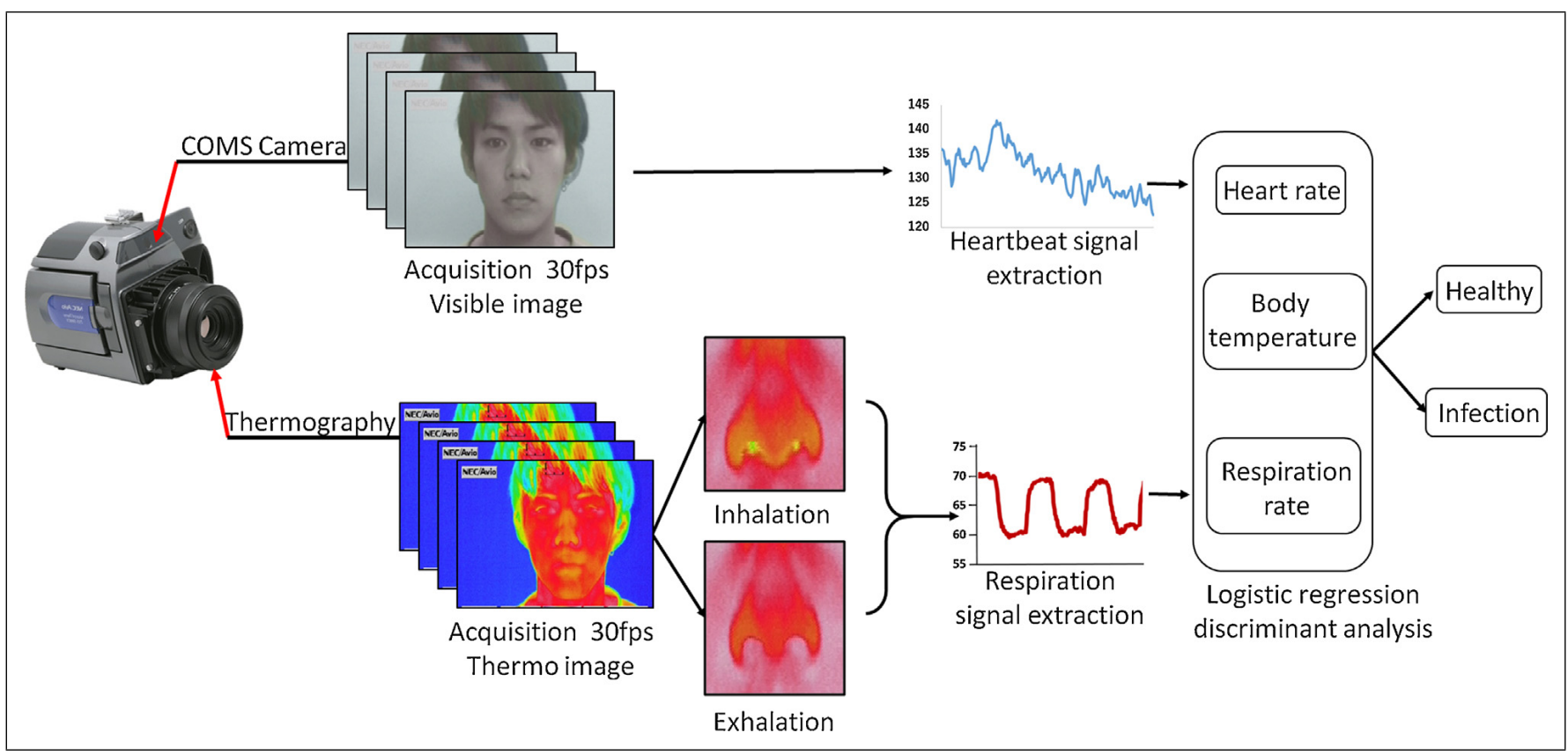

FIGURE 55: Contactless vital sign measurement system using a COMS camera [62].

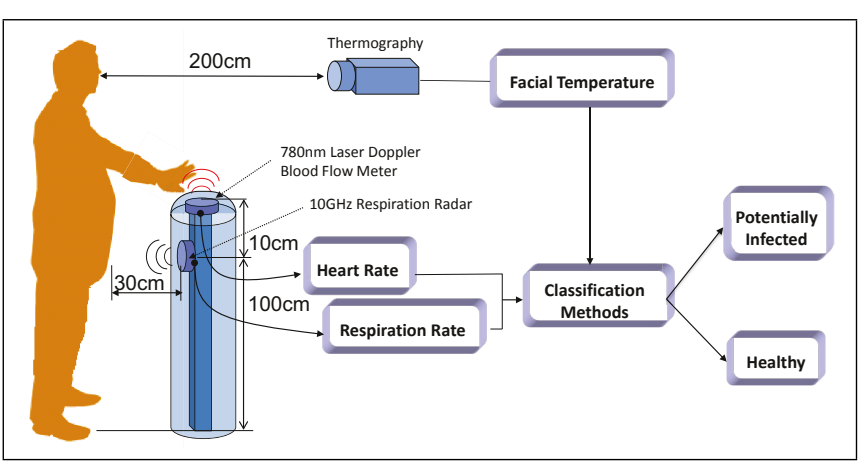

FIGURE 56: Contactless vital sign measurement system [63].

ing technology to be applied in many different areas [73] [74].

According to [75], Wi-Fi sensing technology progress has been impeded by three main obstacles. The first is the unknown baseband design and its influence on CSI. The second is the lack of access to low-level control hardware and the third is the lack of a flexible and versatile software for hardware control. The authors address each of this problem in their work and propose PicoScenes, which is a Wi-Fi sensing technology that authors claim can greatly facilitate the research on Wi-Fi sensing by giving direct access to features of QCA9300 and IWL5300.

Authors in [76] concur that health monitoring using wireless sensors is a popular topic but with open problems, hence, numerous solutions entailing wearable, wireless, open-source, and noninvasive techniques have been proposed. Moreover, authors claim that most of the available platforms for developing the wireless sensing technology are limited and lack flexibility. Therefore, authors propose a new open hardware architecture used to design sensor nodes utilized in healthcare. In addition, authors develop a simulation tool which facilitates the connections with the hardware and simplifies complex systems.

Authors in [77] and [78] acknowledge the high demand for wireless sensor networks in areas such as medicine, military, and structural and environmental monitoring. However, according to the authors, most of the existing platforms for developing these sensor networks are not power efficient. Therefore, authors review some of the most recent and ultra low power processors available in literature. Examples of the hardware systems discussed include general purpose commodity-based systems, smart dust-early event driven, sub-threshold systems, asynchronous-SNAP, charm-network stack acceleration, and Harvard eventdriven architecture.

Other hardware platforms that facilitate wireless sensing research include: Mica2 [79], Mica2 Dot [79], MicaZ [79], IRIS [79], Cricket v1 and v2 [80], Eyes [81], EyesIFX v1 and v2 [82], BTnode [83], Telos B [84], Tmote Sky [85], V-Link [86], and NI WSN-3202 [87],

\section{CHALLENGES}

In this section, we analyse the challenges faced by the above mentioned contactless sensing technologies.

\section{A. CHALLENGES FACING FMCW-BASED SENSING}

FMCW-based sensing technique discussed in details in the previous sections, is a method where a continues frequency modulated wave and its reflections' characteristics are stud- 


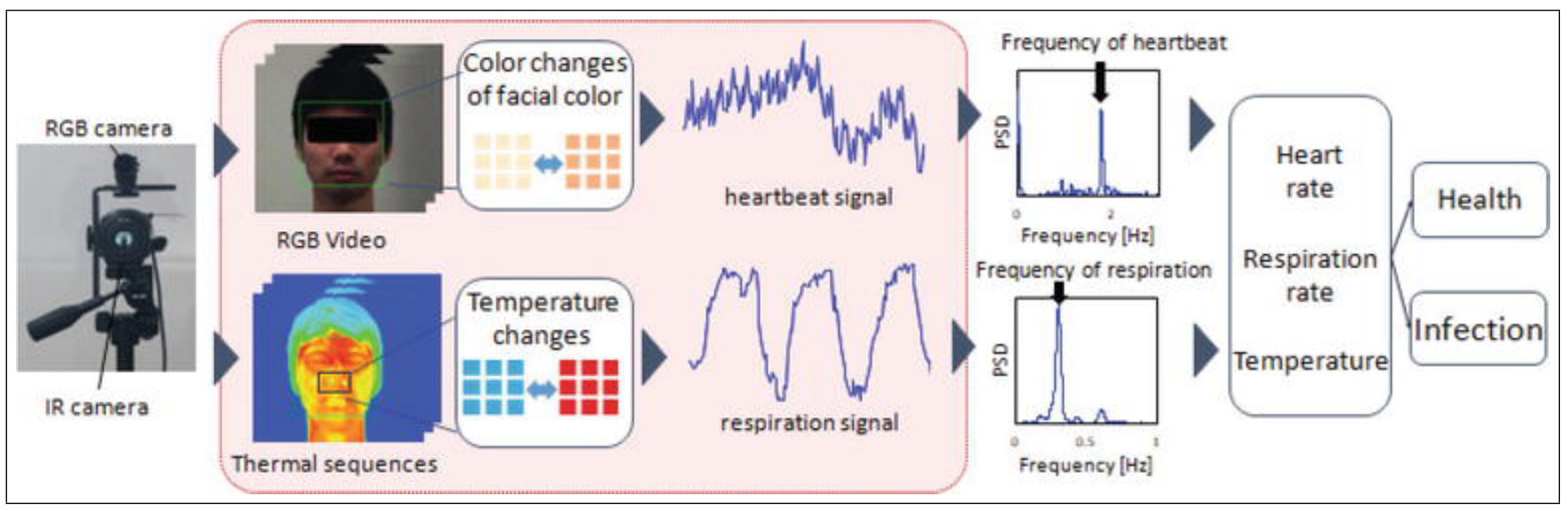

FIGURE 57: Infectious decease screening system using infrared and RGB imaging [70].

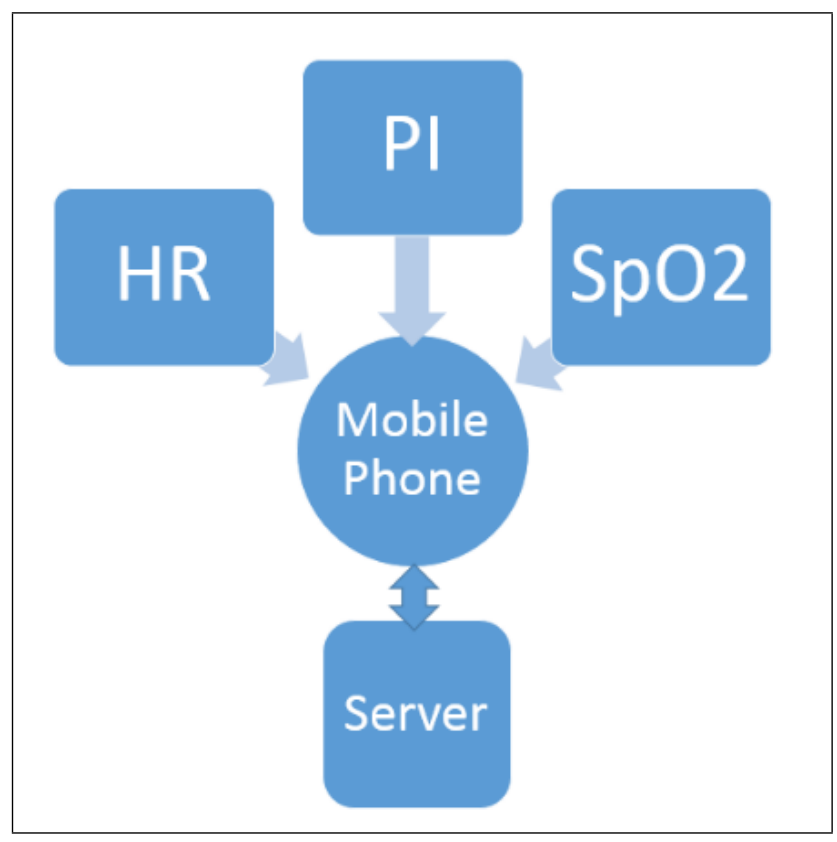

FIGURE 58: Proposed smart phone camera vital signs measurement system model [72].

ied. The changes of the wave after reflection convey data that can be decoded to sense information about a user such as motion, heartbeat rate, sleep, and other emotions without using body contact sensors. Some challenges encountered when using this technique are as follows:

1) Bandwidth: The range resolution of FMCW is constrained by bandwidth. Higher resolutions means higher bandwidth [88].

2) Power consumption: FMCW radar systems are able to provide fine range and Doppler information, nevertheless, the power consumption of these system is high [12].

3) Orientation: Since the FMCW-based system sends a continues signal in a given direction, the orientation of the subject is a factor where for example if the chest is being observed for respiratory monitoring some critical signals may be missed if the patient's thorax is not facing the antenna because the signal is weak [34].

4) Accuracy: Accuracy is one of the most important attribute for a vital signs monitoring system in an AAL environment for instance. In the FMCW-based radar set-up, the FFT is used to measure the range or property of the detected subject. Nevertheless, FFT is not always accurate and accuracy-improving algorithms need to be utilized to enhance the precision of the system [89]. Some signal processing algorithms used are windowing, zero-padding, chirp-z transform, and frequency estimators.

5) Noise and Cost: Authors in [26] mention that the main problem that persisted in their design of the Doppler radar vital sign monitoring system is finding ways to minimize the background noise during motion while maintaining low cost.

6) Interference: Authors in [28] note that interference from things such as moving fans and neighbors is one of the biggest challenge faced by the design of EZSleep used to detect insomnia. This is because the RF signal provided to the classifier can easily reflect the motion from the interference.

\section{B. CHALLENGES FACING CSI-BASED SENSING}

In this section, we look at the different challenges faced by CSI-based systems discussed in section III B. CSI contains tremendous data about the environment, this data can be extracted to create new information. Nevertheless, there are numerous challenges faced by CSI-based systems such as:

1) Motion: Authors in [46] proposed a contactless respiration monitoring system using RSS. Moreover, the authors discuss further research issues such as the limits of distinguishing between subject's body motion and actual breathing motion. Body motion such as arm or body movement occupies most of the sensing frequency, therefore, the main research question posed by [46] is to investigate weather some of the 
frequency channels remain unaffected despite the body motion interference. Moreover, in a Wi-Fi CSI-based gesture recognition system the main problem was that the gesture movements produced minute Doppler shift changes which was very hard to detect [47, 90].

2) Subject's characteristics: In breathing monitoring using RSS, the characteristics of the patient such as age, gender, and size should be taken into consideration. For instance, the chest movement of a child is considerably faster than that of an adult, hence, it is expected that monitoring a infant's chest movement is more challenging. This factors must be taken into consideration when designing CSI-based respiratory monitoring system [46].

3) Unreliability: According to [90], RSS provides only a rough estimate about the information of a wireless channel and does not provide detailed information about the effect of multipath. Moreover, RSS measurements are single values per packet which represent SINR over the channel.

4) Cost: In large crowd sensing application, high training cost is the main limiting factor in CSI-based technique, moreover, it is very difficult to get the actual ground number when a large group of people is involved [90].

5) Range: Wi-Fi-based sensing techniques have limited range of detection compared to other techniques such as ulta-wide band (UWB), hence, this could limit their application [48, 90].

\section{CHALLENGES FACING WSN-BASED SENSING}

The proposed technique used by [57] uses ultra low-power radio-frequency transceiver to detect the motion and breathing of the patient during sleep. Some challenges faced by this scheme are as follows:

1) Security: It is critical that the information of a patient is protected. The sensed signal from the patient should be secure and with limited access. Moreover, the the sensed signal from one patient should not be mistaken for another [91, 92].

2) Reliability: The system must be very reliable. Sensing and wireless communications errors must be reduced because an undetected signal could lead to fatal consequences.

3) Intelligence: Intelligent systems can also be integrated to the WSN paradigm to equip the sensors with the ability to continuously monitor the patient or perform an event driven surveillance.

4) Transmission power: The transmit power used by the sensors should be very low to prevent interference and radiation [93, 92], which could be harmful to a person. However, the power should also be enough to provide power for sensing. Potential solutions for the power problem in WSN have been proposed in [91].

5) Mobility: The communication path used for one data transfer is outdated in case of mobile WSN nodes. This means extra processing power needed to compute new routs as well as extended delays.

6) Cost: Expenditure is incurred by the routing algorithm in terms of bandwidth, delay, jitter, among other performance metrics. Based on the requirements of the system, a trade-off is needed between cost, QoS and resources. Moreover, sustaining a route table is also costly [94]. In addition, WSN have limited power, computation, and memory.

7) Delay: For real-time monitoring systems, delay is one of the most critical factor and hence, must be minimized. Delay can be caused by network characteristics such as node mobility, interrupted connection, and rerouting [94].

8) Bandwidth: In WSN bandwidth is a limited resource and systems must carefully select routes to utilize the available bandwidth while maintaining system performance [94].

\section{CHALLENGES FACING CAMERA-BASED SENSING}

Even though the use of cameras for persons monitoring such as fall detection is well researched, there are still some technological challenges to overcome, such as:

1) Sensitivity: In the case of the use of thermal cameras for instance, infrared thermography (IRT) which was a popular mechanism used to detect patients carrying infectious diseases suffers from low sensitivity and specificity because monitoring high temperature alone is not sufficient to detecting an infected patient [24].

2) Occlusion: If there are many obstacles such as furniture in a room, there might be a need to add more cameras [95].

3) Acceptance: Many subjects are very reluctant to accept this form of sensing in their homes or around them. They user usually feels like they will loose their privacy [95].

\section{LESSONS LEARNED AND RESEARCH DIRECTION}

In this section we summarise the lessons learned from this survey as well as the future expected direction of research in this field.

After presenting the contactless technology to supervise human vital signs with the different techniques and systems classified as FMCW-radar, channel state information, wireless sensor network, and camera based, we summarize and compare these techniques according to supervised vital signs, used technique, and the used band as shown in Table (1), as well as according to the machine learning technique used for classification as shown in Table (2).

Table (1) shows that FMCW is the most exploited technology to develop contactless sensing devices in medicine. This study has also shown that contantless sensors have great potential to revolutionise human vital sign detection and monitoring. The study has demonstrated that contactless sensing technology can be used to limit contact between patients, 
TABLE 1: Comparison according to the monitored vital sign, techniques, and Bands

\begin{tabular}{|c|c|c|c|}
\hline Approaches & Vital sign & Technique & Band (GHz) \\
\hline 11 & $\begin{array}{l}\text { Breathing and heart- } \\
\text { beat }\end{array}$ & FMCW & 77 \\
\hline $\mid 27$ & Motion, HR, RR & FMCW & 2.4 \\
\hline EQ-Radio[43] & Emotion & FMCW & $5.46-7.25$ \\
\hline EZ-Sleep 28 & $\begin{array}{l}\text { Breathing, motion, } \\
\text { sleep }\end{array}$ & FMCW & $5.46-7.25$ \\
\hline Vital-Radio[29] & Breathing, HR & FMCW & $5.46-7.25$ \\
\hline Marko[30] & Behavior & FMCW & $5.46-7.24$ \\
\hline Witrack| 44 & Localization & FMCW & $5.56-7.25$ \\
\hline WiTrack2.0 [45] & Localization & FMCW & $5.46-7.25$ \\
\hline Aryokee 31$]$ & Fall & FMCW & $5.46-7.24$ \\
\hline |32| & sleep & FMCW & $\begin{array}{l}\text { Not } \\
\text { specified }\end{array}$ \\
\hline WiGait[33] & $\begin{array}{l}\text { Gait velocity, Stride } \\
\text { length }\end{array}$ & FMCW & $5.46-7.24$ \\
\hline Emirald[36] & FSHD & FMCW & Not \\
\hline Emirald 37 & $\begin{array}{l}\text { Surgery recovery (en- } \\
\text { dometriosis) }\end{array}$ & FMCW & Not \\
\hline 38 & Head position & FMCW & \\
\hline $\begin{array}{l}\text { BodyCompass } \\
{[41]}\end{array}$ & Sleep position & FMCW & $\begin{array}{l}\text { specifled } \\
5.4 \mathrm{GHz} \quad- \\
7.2 \mathrm{GHz}\end{array}$ \\
\hline Emirald[39] & Pacing & FMCW & $\begin{array}{l}\text { Not } \\
\text { specified }\end{array}$ \\
\hline 42 & PD & FMCW & $\begin{array}{l}\text { Not } \\
\text { specified }\end{array}$ \\
\hline [40] & Human Figure & FMCW & $5.46-7.24$ \\
\hline 34 & $\begin{array}{l}\text { Respiratory rate and } \\
\text { heartbeat }\end{array}$ & FMCW & 5.8 \\
\hline 35 & Respiration rate & FMCW & 5.8 \\
\hline 17 & $\begin{array}{l}\text { Human activity (sit- } \\
\text { ting standing) }\end{array}$ & CSI & 2.4 \\
\hline$|46|$ & $\mathrm{RR}$ & CSI & 2.4 \\
\hline WIDETECT[47] & Motion & CSI & 5.805 \\
\hline WifiU [48] & Gait & CSI & 5 \\
\hline WIFID 49] & Identification & CSI & $\begin{array}{l}\text { Not } \\
\text { specified }\end{array}$ \\
\hline WiSee 56] & Gesture Recognition & CSI & 5 \\
\hline [53] & Human activity & CSI & 5.8 \\
\hline & Gesture Recognition & CSI & 5 \\
\hline & Heart beat & CSI & $24 \mathrm{GHz}$ \\
\hline |D-CNN|50| & Paraparesis & CSI & 5.32 \\
\hline [51] & $\begin{array}{l}\text { Human activity (sit- } \\
\text { ting standing) }\end{array}$ & CSI & 3.3 \\
\hline [52] & Human posture & CSI & 3.198 \\
\hline SleepMinder [57] & $\begin{array}{l}\text { Sleep(AHI), Breath- } \\
\text { ing }\end{array}$ & WSN & 5.8 \\
\hline 60 & $\begin{array}{l}\text { Localisation, breath- } \\
\text { ing }\end{array}$ & WSN & 2.4 \\
\hline 59 & $\begin{array}{l}\text { Temperature, } \\
\text { luminosity }\end{array}$ & WSN & 2.4 \\
\hline 24 & Blood Volume & Camera & $0.75-4.0 \mathrm{~Hz}$ \\
\hline 63 & $\begin{array}{l}\text { Heart rate, Tempera- } \\
\text { ture }\end{array}$ & Camera & $10 \mathrm{GHz}$ \\
\hline & See through wall & Camera & $10 \mathrm{GHz}$ \\
\hline \begin{tabular}{|l|l|}
70 & 71 \\
\end{tabular} & $\begin{array}{l}\text { Respiration and } \\
\text { heartbeat }\end{array}$ & Camera & $\begin{array}{l}\text { Not } \\
\text { specified }\end{array}$ \\
\hline 72 & $\begin{array}{l}\text { Heart rate, per-fusion } \\
\text { index and oxygen sat- } \\
\text { uration }\end{array}$ & Camera & $\begin{array}{l}\text { Not } \\
\text { specified }\end{array}$ \\
\hline
\end{tabular}

TABLE 2: Comparison according to the classification tool.

\begin{tabular}{|c|c|}
\hline Approaches & Classification tool \\
\hline$[26$ & Not specified \\
\hline |27] & Not specified \\
\hline EQ-Radio[43] & SVM \\
\hline EZ-Sleep $|28|$ & CNN \\
\hline Emirald|39] & Not specified \\
\hline Vital-Radio|29] & Not specified \\
\hline Marko[30] & $\mathrm{NN}$ \\
\hline Witrack|44] & Not specified \\
\hline WiTrack2.0[45] & Not specified \\
\hline Aryokee 31 & SVM, NN \\
\hline [32] — & NN \\
\hline WiGait[33] & Not specified \\
\hline [40] ए & Not specified \\
\hline 46 & HMM \\
\hline WIDETECT[47| & Not specified \\
\hline WifiU[48] & SVM \\
\hline WIFID|49| & SVM \\
\hline WiSee/56 & Not specified \\
\hline [53] ए & KNN \\
\hline $1 \mathrm{D}-\mathrm{CNN} \mid 50]$ & $\mathrm{CNN}$ \\
\hline Sleep Minder|57] & Not specified \\
\hline$[60]$ & Not specified \\
\hline
\end{tabular}

doctors, and other people and hence preventing the spread of highly contagious diseases such as Covid-19. However, the spread and persistence of Covid-19 pandemic in the year 2020 clearly showed that contactless sensors have not been developed and deployed enough. As a result, researchers and engineers have realized this gap and contactless sensing is expected to be given more attention leading to development of advanced contactless sensing systems in the near future.

The future direction of this work will be to analyse the most effective method used to perfect the highlighted challenges in the previous sections for each contactless sensing scheme.

\section{CONCLUSION}

Vital signs monitoring is a necessary, useful, and lifesaving method used in healthcare to monitor and follow the subject's well-being and trigger the required intervention, especially in high-risk scenarios. contactless sensor technology is a new technology applied in medicine that ensures that the monitoring of a patient is done in a remote manner with no patient-healthcare provider physical contact and without asking the patient to wear any body-contact sensors. In this survey, we studied the current approaches and techniques used to monitor human vital signs using contactless (devicefree or sensorless) wireless technology. Our analysis yields the following results: contactless sensing is a viable sensing technology in the field of medicine with the ability to save human life and provide valuable data while ensuring comfort to the subject since no direct contact is required. In addition, utilizing contactless sensor technology will reduce direct and physical interactions between the patient and the care giver, hence lowering the chances of spreading contagious diseases. Moreover, existing techniques are modeled around FMCWradar (which is a widely used technique as the basis of other sensing methods), channel state information, wireless 
sensor networks, and camera. Also, we discussed some of the main challenges faced by each of the contactless sensing techniques as well as enabling hardware technologies.

\section{References}

[1] US Census Bureau. International database. Table 094. Midyear population, by age and sex. 2008.

[2] Sahdeo Prasad, Bokyung Sung, and Bharat B Aggarwal. "Age-associated chronic diseases require age-old medicine: role of chronic inflammation". In: Preventive medicine 54 (2012), S29-S37.

[3] Benoit Latré et al. "A survey on wireless body area networks". In: Wireless networks 17.1 (2011), pp. 118.

[4] Abdullah M Al-Awadhi et al. "Death and contagious infectious diseases: Impact of the COVID-19 virus on stock market returns". In: Journal of Behavioral and Experimental Finance (2020), p. 100326.

[5] Xiaochen Lai et al. "A survey of body sensor networks". In: Sensors 13.5 (2013), pp. 5406-5447.

[6] Jehad M Hamamreh, Haji M Furqan, and Huseyin Arslan. "Classifications and applications of physical layer security techniques for confidentiality: A comprehensive survey". In: IEEE Communications Surveys \& Tutorials 21.2 (2018), pp. 1773-1828.

[7] O Boric-Lubeke and Victor M Lubecke. "Wireless house calls: using communications technology for health care and monitoring". In: IEEE Microwave Magazine 3.3 (2002), pp. 43-48.

[8] Mari Zakrzewski, Arto Kolinummi, and Jukka Vanhala. "Contactless and unobtrusive measurement of heart rate in home environment". In: 2006 International Conference of the IEEE Engineering in Medicine and Biology Society. IEEE. 2006, pp. 20602063.

[9] Luis A Nunes Amaral et al. "Scale-independent measures and pathologic cardiac dynamics". In: Physical Review Letters 81.11 (1998), p. 2388.

[10] Anders Høst-Madsen et al. "Signal processing methods for Doppler radar heart rate monitoring". In: Signal processing techniques for knowledge extraction and information fusion. Springer, 2008, pp. 121-140.

[11] Yong Wang et al. "Remote Monitoring of Human Vital Signs Based on 77-GHz mm-Wave FMCW Radar". In: Sensors 20.10 (2020), p. 2999.

[12] Mamady Kebe et al. "Human Vital Signs Detection Methods and Potential Using Radars: A Review". In: Sensors 20.5 (2020), p. 1454.

[13] Lukas Piotrowsky et al. "Enabling High Accuracy Distance Measurements With FMCW Radar Sensors". In: IEEE Transactions on Microwave Theory and Techniques 67.12 (Dec. 2019), pp. 5360-5371. DOI: 10.1109/tmtt.2019.2930504.

[14] Pavlo Molchanov et al. "Short-range FMCW monopulse radar for hand-gesture sensing". In: 2015
IEEE Radar Conference (RadarCon). IEEE, May 2015. DOI: 10.1109/radar.2015.7131232

[15] Sandeep Rao. "Introduction to mmWave sensing: FMCW radars". In: Texas Instruments (TI) mmWave Training Series (2017).

[16] Bong-seok Kim et al. "Low-complexity joint range and doppler FMCW radar algorithm based on number of targets". In: Sensors 20.1 (2020), p. 51.

[17] Bo Tan et al. "Exploiting WiFi Channel State Information for Residential Healthcare Informatics". In: IEEE Communications Magazine 56.5 (May 2018), pp. 130-137. DOI: $10.1109 /$ mcom.2018.1700064

[18] Yongsen Ma, Gang Zhou, and Shuangquan Wang. "WiFi Sensing with Channel State Information". In: ACM Computing Surveys 52.3 (July 2019), pp. 1-36. DOI: $10.1145 / 3310194$

[19] Suraiya Tarannum and Shaista Farheen. "Wireless Sensor Networks for Healthcare Monitoring: A Review". In: Inventive Computation Technologies. Springer International Publishing, Nov. 2019, pp. 669-676. DOI: 10.1007/978-3-030-33846-6_72.

[20] Bassam Al-Shargabi. "Performance of RPL in Healthcare Wireless Sensor Network". In: International Journal of Emerging Trends in Engineering Research 8.3 (Mar. 2020), pp. 797-803. DOI: 10.30534/ijeter/ 2020/31832020.

[21] Victor Shnayder et al. "Sensor networks for medical care". In: Proceedings of the 3rd international conference on Embedded networked sensor systems SenSys '05. ACM Press, 2005. DOI: 10.1145/1098918. 1098979 .

[22] Jeonggil Ko et al. "MEDiSN". In: ACM Transactions on Embedded Computing Systems 10.1 (Aug. 2010), pp. 1-29. DOI: $10.1145 / 1814539.1814550$

[23] Octav Chipara et al. "Reliable clinical monitoring using wireless sensor networks". In: Proceedings of the 8th ACM Conference on Embedded Networked Sensor Systems - SenSys '10. ACM Press, 2010. DOI: 10.1145/1869983.1869999.

[24] Toshiaki Negishi et al. "Contactless Vital Signs Measurement System Using RGB-Thermal Image Sensors and Its Clinical Screening Test on Patients with Seasonal Influenza". In: Sensors 20.8 (Apr. 2020), p. 2171. DOI: $10.3390 / \mathrm{s} 20082171$.

[25] Halgurd S Maghdid et al. "A novel ai-enabled framework to diagnose coronavirus covid 19 using smartphone embedded sensors: Design study". In: arXiv preprint arXiv:2003.07434 (2020).

[26] Rich Fletcher and Jing Han. "Low-cost differential front-end for Doppler radar vital sign monitoring". In: 2009 IEEE MTT-S International Microwave Symposium Digest. IEEE. 2009, pp. 1325-1328.

[27] Amy D Droitcour, Olga Boric-Lubecke, and Gregory TA Kovacs. "Signal-to-noise ratio in Doppler radar system for heart and respiratory rate measurements". 
In: IEEE transactions on microwave theory and techniques 57.10 (2009), pp. 2498-2507.

[28] Chen-Yu Hsu et al. "Zero-effort in-home sleep and insomnia monitoring using radio signals". In: Proceedings of the ACM on Interactive, Mobile, Wearable and Ubiquitous Technologies 1.3 (2017), pp. 1-18.

[29] Fadel Adib et al. "Smart homes that monitor breathing and heart rate". In: Proceedings of the 33rd annual ACM conference on human factors in computing systems. 2015, pp. 837-846.

[30] Chen-Yu Hsu et al. "Enabling identification and behavioral sensing in homes using radio reflections". In: Proceedings of the 2019 CHI Conference on Human Factors in Computing Systems. 2019, pp. 1-13.

[31] Yonglong Tian et al. "RF-based fall monitoring using convolutional neural networks". In: Proceedings of the ACM on Interactive, Mobile, Wearable and Ubiquitous Technologies 2.3 (2018), pp. 1-24.

[32] Mingmin Zhao et al. "Learning sleep stages from radio signals: A conditional adversarial architecture". In: International Conference on Machine Learning. 2017, pp. 4100-4109.

[33] Chen-Yu Hsu et al. "Extracting gait velocity and stride length from surrounding radio signals". In: Proceedings of the 2017 CHI Conference on Human Factors in Computing Systems. 2017, pp. 2116-2126.

[34] Giulia Sacco et al. "An FMCW Radar for Localization and Vital Signs Measurement for Different Chest Orientations". In: Sensors 20.12 (June 2020), p. 3489. DOI: $10.3390 / \mathrm{s} 20123489$.

[35] Lili Xie et al. "Wireless Healthcare System for Life Detection and Vital Sign Monitoring". In: 2020 IEEE 91st Vehicular Technology Conference (VTC2020Spring). IEEE, May 2020. DOI: $10.1109 /$ vtc2020 spring48590.2020.9128431.

[36] Kabelac Z et al. "An In-Home Study of Facioscapulohumeral Muscular Dystrophy (FSHD) Patients using Contactless Wireless Sensing and Machine Learning (1561)". In: (2020).

[37] Megan Loring et al. "Novel Technology to Capture Objective Data from Patients' Recovery from Laparoscopic Endometriosis Surgery". In: Journal of Minimally Invasive Gynecology (June 2020). DOI: 10.1016/ j.jmig.2020.06.011.

[38] Sina Akbarian et al. "Automated Non-Contact Detection of Head and Body Positions During Sleep". In: IEEE Access 7 (2019), pp. 72826-72834. DOI: 10. 1109/access.2019.2920025

[39] Ipsit V. Vahia et al. "Radio Signal Sensing and Signal Processing to Monitor Behavioral Symptoms in Dementia: A Case Study". In: The American Journal of Geriatric Psychiatry 28.8 (Aug. 2020), pp. 820-825. DOI: $10.1016 /$ j.jagp.2020.02.012

[40] Fadel Adib et al. "Capturing the human figure through a wall". In: ACM Transactions on Graphics (TOG) 34.6 (2015), pp. 1-13.
[41] Shichao Yue et al. "BodyCompass". In: Proceedings of the ACM on Interactive, Mobile, Wearable and Ubiquitous Technologies 4.2 (June 2020), pp. 1-25. DOI: $10.1145 / 3397311$

[42] Zachary Kabelac et al. "Passive Monitoring at Home: A Pilot Study in Parkinson Disease". In: Digital Biomarkers 3.1 (Apr. 2019), pp. 22-30. DOI:10.1159/ 000498922

[43] Mingmin Zhao, Fadel Adib, and Dina Katabi. "Emotion recognition using wireless signals". In: Proceedings of the 22nd Annual International Conference on Mobile Computing and Networking. 2016, pp. 95-108.

[44] Fadel Adib et al. "3D tracking via body radio reflections". In: 11th \{USENIX\} Symposium on Networked Systems Design and Implementation ( $\{N S D I\} 14)$. 2014, pp. 317-329.

[45] Fadel Adib, Zachary Kabelac, and Dina Katabi. "Multi-person localization via $\{\mathrm{RF}\}$ body reflections". In: 12th $\{$ USENIX $\}$ Symposium on Networked Systems Design and Implementation (\{NSDI\} 15). 2015, pp. 279-292.

[46] Ossi Kaltiokallio et al. "Non-invasive respiration rate monitoring using a single COTS TX-RX pair". In: IPSN-14 Proceedings of the 13th International Symposium on Information Processing in Sensor Networks. IEEE. 2014, pp. 59-69.

[47] Feng Zhang et al. "Widetect: A robust and lowcomplexity wireless motion detector". In: 2018 IEEE International Conference on Acoustics, Speech and Signal Processing (ICASSP). IEEE. 2018, pp. 63986402.

[48] Wei Wang, Alex X Liu, and Muhammad Shahzad. "Gait recognition using wifi signals". In: Proceedings of the 2016 ACM International Joint Conference on Pervasive and Ubiquitous Computing. 2016, pp. 363373.

[49] Feng Hong et al. "WFID: Passive device-free human identification using WiFi signal". In: Proceedings of the 13th International Conference on Mobile and Ubiquitous Systems: Computing, Networking and Services. 2016, pp. 47-56.

[50] Lei Guan et al. "A non-contact paraparesis detection technique based on 1D-CNN". In: IEEE Access 7 (2019), pp. 182280-182288.

[51] Neena Damodaran et al. "Device free human activity and fall recognition using WiFi channel state information (CSI)". In: CCF Transactions on Pervasive Computing and Interaction 2.1 (Jan. 2020), pp. 1-17. DOI: $10.1007 / \mathrm{s} 42486-020-00027-1$

[52] Jingzhi Hu et al. "Reconfigurable Intelligent Surface Based RF Sensing: Design, Optimization, and Implementation". In: IEEE Journal on Selected Areas in Communications 38.11 (Nov. 2020), pp. 2700-2716. DOI: $10.1109 /$ jsac.2020.3007041. 
[53] Qinyi Xu et al. "Indoor events monitoring using channel state information time series". In: IEEE Internet of Things Journal 6.3 (2019), pp. 4977-4990.

[54] Youwei Zeng et al. "MultiSense". In: Proceedings of the ACM on Interactive, Mobile, Wearable and Ubiquitous Technologies 4.3 (Sept. 2020), pp. 1-29. DOI: $10.1145 / 3411816$.

[55] Nebojša Malešević et al. "Contactless Real-Time Heartbeat Detection via $24 \mathrm{GHz}$ Continuous-Wave Doppler Radar Using Artificial Neural Networks". In: Sensors 20.8 (Apr. 2020), p. 2351. DOI: $10.3390 /$ s20082351.

[56] Qifan Pu et al. "Whole-home gesture recognition using wireless signals". In: Proceedings of the 19th annual international conference on Mobile computing \& networking. 2013, pp. 27-38.

[57] Alberto Zaffaroni et al. "SleepMinder: an innovative contact-free device for the estimation of the apnoeahypopnoea index". In: 2009 annual international conference of the IEEE engineering in medicine and biology society. IEEE. 2009, pp. 7091-9094.

[58] Niall A Fox et al. "An evaluation of a non-contact biomotion sensor with actimetry". In: 2007 29th Annual International Conference of the IEEE Engineering in Medicine and Biology Society. IEEE. 2007, pp. 2664-2668.

[59] Nouha Sghaier et al. "Wireless Sensor Networks for medical care services". In: 2011 7th International Wireless Communications and Mobile Computing Conference. IEEE, July 2011. DOI: 10 . 1109/ iwcmc.2011.5982596.

[60] Neal Patwari et al. "Breathfinding: A wireless network that monitors and locates breathing in a home". In: IEEE Journal of Selected Topics in Signal Processing 8.1 (2013), pp. 30-42.

[61] George Vasilev Angelov et al. "Healthcare Sensing and Monitoring". In: Lecture Notes in Computer Science. Springer International Publishing, 2019, pp. 226-262. DOI: 10.1007/978-3-030-10752-9_10

[62] Guanghao Sun et al. "Remote sensing of multiple vital signs using a CMOS camera-equipped infrared thermography system and its clinical application in rapidly screening patients with suspected infectious diseases". In: International Journal of Infectious Diseases 55 (Feb. 2017), pp. 113-117. DOI: $10.1016 /$ j.ijid.2017. 01.007 .

[63] Yu Yao et al. "Multiple Vital-Sign-Based Infection Screening Outperforms Thermography Independent of the Classification Algorithm". In: IEEE Transactions on Biomedical Engineering 63.5 (May 2016), pp. 1025-1033. DOI: $10.1109 /$ tbme.2015.2479716.

[64] Eddie Y.K Ng, G.J.L Kawb, and W.M Chang. "Analysis of IR thermal imager for mass blind fever screening”. In: Microvascular Research 68.2 (Sept. 2004), pp. 104-109. DOI: 10.1016/j.mvr.2004.05.003
[65] Ming-Fu Chiang et al. "Mass Screening of Suspected Febrile Patients with Remote-sensing Infrared Thermography: Alarm Temperature and Optimal Distance". In: Journal of the Formosan Medical Association 107.12 (Dec. 2008), pp. 937-944. DOI: 10.1016/ s0929-6646(09)60017-6

[66] Guanghao Sun et al. "Applications of Infrared Thermography for Noncontact and Noninvasive Mass Screening of Febrile International Travelers at Airport Quarantine Stations". In: Application of Infrared to Biomedical Sciences. Springer Singapore, 2017, pp. 347-358. DOI: 10.1007/978-981-10-3147-2_19.

[67] "The Application Value of Non-contact Infrared Thermometers in Temperature Screening in the General Population”. In: Case Medical Research (Feb. 2020). DOI: $10.31525 / \mathrm{ct} 1$-nct04274621

[68] Toshiaki Negishi et al. "Stable Contactless Sensing of Vital Signs Using RGB-Thermal Image Fusion System with Facial Tracking for Infection Screening". In: 2018 40th Annual International Conference of the IEEE Engineering in Medicine and Biology Society (EMBC). IEEE, July 2018. DOI: 10.1109/embc.2018. 8513300

[69] Yunqiang Yang and A.E. Fathy. "See-Through-Wall Imaging Using Ultra Wideband Short-Pulse Radar System". In: 2005 IEEE Antennas and Propagation Society International Symposium. IEEE. DOI: 10 . 1109/aps.2005.1552508

[70] Guanghao Sun et al. "Noncontact Monitoring of Vital Signs with RGB and Infrared Camera and Its Application to Screening of Potential Infection". In: Non-Invasive Diagnostic Methods - Image Processing. IntechOpen, Dec. 2018. DOI: $10.5772 /$ intechopen . 80652

[71] Christoph Hoog Antink et al. "A Broader Look: Camera-Based Vital Sign Estimation across the Spectrum". In: Yearbook of Medical Informatics 28.01 (Aug. 2019), pp. 102-114. DOI: $10.1055 /$ s-00391677914.

[72] Mohammad Adibuzzaman, Sheikh Iqbal Ahamed, and Richard Love. "A personalized model for monitoring vital signs using camera of the smart phone". In: Proceedings of the 29th Annual ACM Symposium on Applied Computing - SAC '14. ACM Press, 2014. DOI: $10.1145 / 2554850.2555019$

[73] Jian Liu et al. "Wireless sensing for human activity: A survey". In: IEEE Communications Surveys \& Tutorials (2019).

[74] Fatma Karray et al. "A comprehensive survey on wireless sensor node hardware platforms". In: Computer Networks 144 (2018), pp. 89-110.

[75] Zhiping Jiang et al. "Eliminating the Barriers: Demystify Wi-Fi Baseband Design And Introduce PicoScenes Wi-Fi Sensing Platform". In: arXiv preprint arXiv:2010.10233 (2020). 
[76] Lucio Ciabattoni et al. "An open and modular hardware node for wireless sensor and body area networks". In: Journal of Sensors 2016 (2016).

[77] Mark Hempstead et al. "Survey of hardware systems for wireless sensor networks". In: Journal of Low Power Electronics 4.1 (2008), pp. 11-20.

[78] Manish Karani, Ajinkya Kale, and Animesh Kopekar. "Wireless sensor network hardware platforms and multi-channel communication protocols: A survey". In: Proceedings on 2nd National Conference on Information and Communication Technology, New York, NY, USA. 2011, pp. 20-23.

[79] Crossbow Technology. MPR-MIB Users Manual. 2004. URL: http://www-db.ics.uci.edu/pages/research/ quasar/MPR - MIB \% 5C \% 20Series \% 5C \% 20User \% 5C \% 20Manual \% 5C \% 207430 - 0021 - 06 _ A . pdf (visited on 2020).

[80] Nissanka B. Priyantha. Cricket. 2006. URL: http:// cricket.csail.mit.edu/ (visited on 2020).

[81] Lodewijk FW Van Hoesel et al. "Design of a lowpower testbed for wireless sensor networks and verification". In: Centre for Telematics and Information Technology de l'université de Twente, Pays-Bas (2003).

[82] Ozgur B Akan, Osman B Karli, and Ozgur Ergul. "Cognitive radio sensor networks". In: IEEE network 23.4 (2009), pp. 34-40.

[83] Jan Beutel, Oliver Kasten, and Matthias Ringwald. "Btnodes-applications and architecture compared". In: Proc. 1st GI/ITG KuVS Fachgespräch Drahtlose Sensornetze (2003), pp. 34-37.

[84] Crossbow. TELOSB. URL: https://www.willow.co.uk/ TelosB_Datasheet.pdf (visited on 2020).

[85] Wireless Sensor Networks. Tmote sky. URL: https:// wirelesssensornetworks.weebly.com/blog/tmote-sky (visited on 2020).

[86] Parker Lord. V-LINK-200. URL: https : / / www . microstrain . com / wireless / v - link - 200 (visited on 2020).

[87] NI. NI WSN-3202. URL: https://wWw.ni.com/entr/support/model.wsn-3202.html (visited on 2020).

[88] M.L. Lees. "Digital beamforming calibration for FMCW radar". In: IEEE Transactions on Aerospace and Electronic Systems 25.2 (Mar. 1989), pp. 281284. DOI: $10.1109 / 7.18690$

[89] Aamna Al Teneiji et al. "Improving the Detection Accuracy of Frequency Modulated Continuous Wave Radar". In: 2018 International Conference on Signal Processing and Information Security (ICSPIS). IEEE, Nov. 2018. DOI: 10.1109/cspis.2018.8642764

[90] Abdullah Khalili et al. "Wi-Fi sensing: applications and challenges". In: The Journal of Engineering 2020.3 (Mar. 2020), pp. 87-97. DOI: 10.1049/joe. 2019.0790 .

[91] C Enz, Nicola Scolari, and Uroschanit Yodprasit. "Ultra low-power radio design for wireless sensor net- works". In: 2005 IEEE International Wkshp on RadioFrequency Integration Technology: Integrated Circuits for Wideband Comm \& Wireless Sensor Networks. IEEE. 2005, pp. 1-17.

[92] Tarun Bala et al. "A survey: issues and challenges in wireless sensor network". In: International Journal of Engineering \& Technology 7.2.4 (Mar. 2018), p. 53. DOI: $10.14419 /$ ijet.v7i2.4.10041

[93] Li Huang et al. "Ultra-low power sensor design for wireless body area networks: challenges, potential solutions, and applications". In: International Journal of Digital Content Technology and its Applications 3.3 (2009), pp. 136-148.

[94] Nishi Gupta, Shikha Gupta, and Satbir Jain. "Efficient Data Transmission in WSN: Techniques and Future Challenges". In: Towards Extensible and Adaptable Methods in Computing. Springer Singapore, 2018, pp. 119-129. DOI:10.1007/978-981-13-2348-5_10.

[95] Ramn Ruiz, Francisco J., and Juan Zapat. "Wireless Sensor Network for Ambient Assisted Living". In: Wireless Sensor Networks: Application-Centric Design. InTech, Dec. 2010. DOI: $10.5772 / 13005$

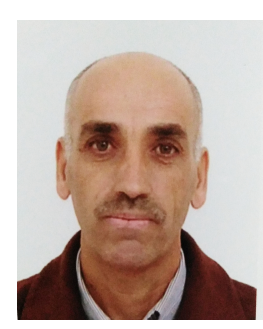

MOHAMED BAHACHE received the BAC in mathematics from Cherif Idrissi in 1989, and the engineering degree in computer science from Constantine University, and received the magister diploma in mobile networking from the University of Laghouat-Algeria in 2015. He was a visiting researcher at Antalya Bilim University-Turkey during Fall, 2019. Currently, he is an assistant professor at the M'sila University. His current research interest includes WBANS and wireless sensing.

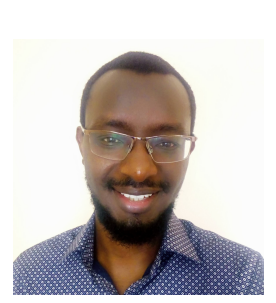

JOEL P. LEMAYIAN received the B.Sc. degree in electrical and electronics engineering from Middle East Technical University Turkey, in 2017. He is presently pursuing the master's (M.Sc.) degree in electrical and computer engineering. He is currently with Antalya Bilim University, Turkey.

$\mathrm{He}$ has worked as a research assistant in both Middle East Technical University and Antalya Bilim University in IoT lab and Neuroscience lab respectively. He is an author of numerus journals, conference papers and book chapters. His research interests include UAVs, 5G Communication networks, Artificial Intelligence, Machine Learning, and the Internet of Things (IoT) applications. 


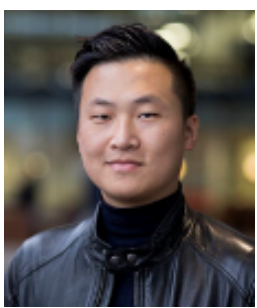

WENJIN WANG received the B.Sc. degree in Biomedical Engineering from Northeastern University, China, in 2011, the M.Sc. degree in Artificial Intelligence from University of Amsterdam, The Netherlands, in 2013, and the Ph.D. degree in Electrical Engineering from Eindhoven University of Technology (TU/e), The Netherlands, in 2017. He is currently an Assistant Professor of Electronic Systems group of TU/e and scientist of Philips Research.

His current research interests include contactless vital signs monitoring, physiological measurement, video health monitoring, and multi-modal sensor fusion for healthcare. He is a guest associate editor for IEEE journals and Sensors.

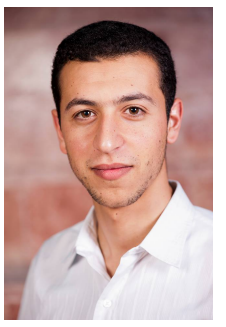

JEHAD M. HAMAMREH received the B.Sc. degree in electrical and telecommunication engineering from An-Najah University, Nablus, in 2013, and the Ph.D. degree in electrical-electronics engineering and cyber systems from Istanbul Medipol University, Turkey, in 2018. He was a Researcher with the Department of Electrical and Computer Engineering, Texas A and M University at Qatar. $\mathrm{He}$ is currently an Assistant Professor with the Electrical and Electronics Engineering Department, Antalya International (Bilim) University, Turkey.

His current research interests include wireless physical and MAC layers security, orthogonal frequency-division multiplexing multiple-input multiple-output systems, advanced waveforms design, multi-dimensional modulation techniques, IoT, 5G \& 6G and orthogonal/non-orthogonal multiple access schemes for future wireless systems. He is a Regular Reviewer for various refereed journals as well as a TPC Member for several international conferences. 